\title{
Mean-Risk Models Using Two Risk Measures: A Multi-Objective Approach
}

\author{
Professor Ken Darby-Dowman \\ Head of the School of Information Systems, Computing and Mathematics \\ Brunel University \\ Uxbridge \\ Middlesex \\ UB8 3PH \\ UK
}

\begin{abstract}
This paper proposes a model for portfolio optimisation, in which distributions are characterised and compared on the basis of three statistics: the expected value, the variance and the CVaR at a specified confidence level. The problem is multi-objective and transformed into a single objective problem in which variance is minimised while constraints are imposed on the expected value and CVaR. In the case of discrete random variables, the problem is a quadratic program. The mean-variance (mean$\mathrm{CVaR}$ ) efficient solutions that are not dominated with respect to CVaR (variance) are particular efficient solutions of the proposed model. In addition, the model has efficient solutions that are discarded by both mean-variance and mean-CVaR models, although they may improve the return distribution. The model is tested on real data drawn from the FTSE 100 index. An analysis of the return distribution of the chosen portfolios is presented.
\end{abstract}

\section{Introduction and motivation}

Mean-risk models are still the most widely used approach in the practice of portfolio selection. With mean-risk models, return distributions are characterized and compared using two statistics: the expected value and the value of a risk measure. Thus, mean-risk models have a ready interpretation of results and in most cases are convenient from a computational point of view. Sceptics on the other hand may question these advantages since the practice of describing a distribution by just two parameters involves great loss of information.

It is evident that the risk measure used plays an important role in making the decisions. Variance was the first risk measure used in mean-risk models (Markowitz 1952) and, in spite of criticism and many proposals of new risk measures (see for example Fishburn (1977), Yitzhaki (1982), Konno and Yamazaki (1991), Ogryczak and Ruszczynski (1999, 2001), Rockafellar and Uryasev 2000, 2002), variance is still the most widely used measure of risk in the practice of portfolio selection. For regulatory and reporting purposes, risk measures concerned with the left tails of distributions (extremely unfavourable outcomes) are used. The most widely used risk measure for such purposes is Value-at-Risk (VaR). However, it is known that VaR has undesirable theoretical properties (it is not subadditive, as shown, for example, in Tasche (2002) and thus fails to reward diversification). In addition, optimisation of VaR leads to a non-convex NP-hard problem which is computationally intractable. In spite of a considerable amount of research, optimising VaR is still an open problem (see for example Larsen et al. 2002, Leyffer et al. 2005 and references therein). For these reasons, another risk measure concerned with the left tail, the Conditional Value-at-Risk (CVaR), is gaining more popularity. CVaR has attractive theoretical properties: it controls the magnitude of losses beyond $\mathrm{VaR}$ and it is coherent (see for example Artzner et al. 1999, Acerbi and Tasche 2002, Tasche 2002, Pflug 2000,

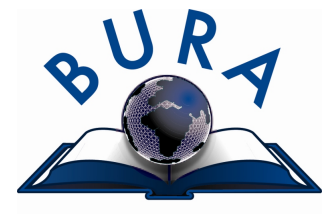


Rockafellar and Uryasev 2002). In addition, CVaR is easy to optimise. Optimising $\mathrm{CVaR}$ is a convex programming problem. In the case when the random variables under consideration are discrete, with a finite number of outcomes, represented by various outcomes under different scenarios, optimising CVaR leads to a linear programming model of finite dimension (Rockafellar and Uryasev 2000, 2002).

Variance and CVaR quantify risk from different perspectives. Variance measures the spread around the expected value of a random variable, while $\mathrm{CVaR}$ measures the expected loss corresponding to a number of worst cases, depending on the chosen confidence level. Thus, the mean-variance and the mean-CVaR models may lead to very different solutions. A portfolio obtained as a solution in the mean-variance model may be considered unacceptable by a regulator, since it may have an excessively large CVaR, leading to big losses under unfavourable scenarios. On the other hand, traditional fund managers may consider a portfolio obtained with the mean-CVaR model unacceptable since it may have an excessively large variance and thus an excessively small Sharpe index (see Luenberger 1998)

In this paper, we seek to address the requirements of the traditional fund manager and the regime imposed by the regulator. We propose a model for portfolio selection that uses both variance and $\mathrm{CVaR}$ in order to make decisions. We call this model the mean-variance-CVaR model. Random variables are described and compared using three statistics: the expected value, variance and CVaR. Thus, the model may be considered as belonging to the family of mean-risk models.

We formally define the preference relation for random variables in this model. The efficient solutions with respect to this preference relation are such that, we cannot improve on one statistic (of the three: expected value, variance and CVaR) without worsening another. Mathematically, the problem is multi-objective (maximise expected return, minimise variance, minimise $\mathrm{CVaR}$ ) and the efficient solutions of the mean-variance-CVaR model are the Pareto optimal solutions of the multi-objective problem.

We prove that the efficient solutions of this model may be found by solving a single objective optimisation problem in which variance is minimised while constraints are imposed on the expected return and the CVaR level. The practical importance of this approach is twofold. Firstly, a solution obtained in this way has an intuitive appeal. For example, if the $\mathrm{CVaR}$ of a mean-variance efficient portfolio is considered as unacceptably large, a constraint could be imposed on the CVaR level and a new portfolio obtained, which has a minimal variance under these conditions. Secondly, the problem is tractable from a computational point of view. In the case where the random variables under consideration are discrete and described by their realisations under various scenarios, the problem is one of quadratic programming.

Generally, the mean-variance and mean-CVaR efficient portfolios are particular efficient solutions of the proposed model ${ }^{1}$. However, most of the efficient portfolios in the mean-variance-CVaR model are dominated in both mean-variance and meanCVaR models, although they may represent improved distributions: a compromise between the classical fund managers' and the regulators' points of view.

The rest of this paper is structured as follows. In section two the portfolio selection problem is described. Section three is concerned with mean-risk models, in

\footnotetext{
${ }^{1}$ There may be a situation when several mean-CVaR efficient portfolios have the same mean return and the same (optimal) CVaR, but different variances. Only the portfolio with the minimal variance is efficient in the proposed model. The same discussion applies for mean-variance efficient portfolios. We reconsider the issue in Section 4.4.
} 
particular with the mean-variance and the mean-CVaR models. In section four we present the mean-variance-CVaR model. Firstly, the preference relation among random variables is defined. The efficient solutions of the proposed model are Pareto non-dominated solutions of a multi-objective problem. Secondly, an optimisation approach for solving the multi-objective problem is proposed. With this approach, the efficient solutions of the proposed model are found by solving a single optimisation problem, in which variance is minimised and constraints are imposed on the expected value and the CVaR level. Thirdly, we describe how all the efficient solutions of the model may be obtained. Finally, the algebraic form of the mean-variance-CVaR model for the case of scenario models is presented. Section five presents the computational results. A dataset, drawn from the FTSE 100 index is used to evaluate the performance of the proposed model. For several fixed levels of expected return, we consider the mean-variance and the mean-CVaR efficient portfolios together with other portfolios, efficient only in the mean-variance-CVaR model. We evaluate their performances using both in-sample and out-of-sample analysis. Section six presents the conclusions.

\section{The portfolio selection problem}

The problem of portfolio selection with one investment period is an example of the general problem of deciding between random variables when larger outcomes are preferred. Decisions are required on the amount (proportion) of capital to be invested in each of a number of available assets such that at the end of the investment period the return is as high as possible. Consider a set of $n$ assets, with asset $j$ in $\{1, \ldots, n\}$ giving a return $R_{j}$ at the end of the investment period. $R_{j}$ is a random variable, since the future price of the asset is not known. Let $x_{j}$ be the proportion of capital invested in asset $\mathrm{j}\left(\mathrm{x}_{\mathrm{j}}=\mathrm{w}_{\mathrm{j}} / \mathrm{w}\right.$ where $\mathrm{w}_{\mathrm{j}}$ is the capital invested in asset $\mathrm{j}$ and $\mathrm{w}$ is the total amount of capital to be invested $)$, and let $\mathrm{x}=\left(\mathrm{x}_{1}, \ldots, \mathrm{x}_{\mathrm{n}}\right)$ represent the portfolio resulting from this choice. This portfolio's return is the random variable:

$\mathrm{R}_{\mathrm{x}}=\mathrm{x}_{1} \mathrm{R}_{1}+\ldots+\mathrm{x}_{\mathrm{n}} \mathrm{R}_{\mathrm{n}}$, with distribution function $F(r)=P\left(R_{x} \leq r\right)$ that depends on the choice $\mathrm{x}=\left(\mathrm{x}_{1}, \ldots, \mathrm{x}_{\mathrm{n}}\right)$.

To represent a portfolio, the weights $\left(\mathrm{x}_{1}, \ldots, \mathrm{x}_{\mathrm{n}}\right)$ must satisfy a set of constraints that forms a feasible set $\mathcal{A}$ of decision vectors. The simplest way to define a feasible set is by the requirement that the weights must sum to 1 and short selling is not allowed. For this basic version of the problem, the set of feasible decision vectors is

$\mathcal{A}=\left\{\left(x_{1}, \ldots, x_{n}\right) / \sum_{j=1}^{n} x_{j}=1, x_{j} \geq 0, \forall j \in\{1, \ldots, n\}\right\}$.

Consider a different portfolio defined by the decision vector $\mathrm{y}=\left(\mathrm{y}_{1}, \ldots, \mathrm{y}_{\mathrm{n}}\right) \in \mathcal{A}$, where $y_{j}$ is the proportion of capital invested in asset $\mathrm{j}$. The return of this portfolio is given by the random variable $\mathrm{R}_{\mathrm{y}}=\mathrm{y}_{1} \mathrm{R}_{1}+\ldots+\mathrm{y}_{\mathrm{n}} \mathrm{R}_{\mathrm{n}}$.

The problem of choosing between portfolio $\mathrm{x}=\left(\mathrm{x}_{1}, \ldots, \mathrm{x}_{\mathrm{n}}\right)$ and portfolio $\mathrm{y}=\left(\mathrm{y}_{1}, \ldots, \mathrm{y}_{\mathrm{n}}\right)$ becomes the problem of choosing between random variables $\mathrm{R}_{\mathrm{x}}$ and $\mathrm{R}_{\mathrm{y}}$. The criteria by which one random variable is considered "better" than another random variable need to be specified and models for choosing between random variables (models for preference) are required. The purpose of such models is firstly, to define a preference relation among random variables and secondly, to identify random variables that are non-dominated with respect to that preference relation. 
The next issue is to consider a practical representation for the random variables that describe asset and portfolio returns. We treat these random variables as discrete and described by realisations under $\mathrm{T}$ states of the world, generated using scenario generation or finite sampling of historical data. For any $i \in\{1, \ldots, T\}$, let state $\omega_{\mathrm{i}}$ occur with probability $\mathrm{p}_{\mathrm{i}}, \sum_{i=1}^{T} p_{i}=1$. Thus, the random returns are defined on a discrete probability space $\{\Omega, \mathcal{F}, \mathrm{P}\}$ with $\Omega=\left\{\omega_{1}, \ldots, \omega_{\mathrm{T}}\right\}, \mathcal{F}$ a $\sigma$-field and $\mathrm{P}\left(\omega_{\mathrm{i}}\right)=\mathrm{p}_{\mathrm{i}}$.

Let $r_{i j}$ be the return of asset $j$ under scenario $i, i \in\{1, \ldots, T\}, j \in\{1, \ldots, n\}$. Thus, the random variable $R_{j}$ representing the return of asset $j$ is finitely distributed over $\left\{\mathrm{r}_{1 \mathrm{j}}, \ldots, \mathrm{r}_{\mathrm{Tj}}\right\}$ with probabilities $\mathrm{p}_{1}, \ldots \mathrm{p}_{\mathrm{T}}$. The random variable $\mathrm{R}_{\mathrm{x}}$ representing the return of portfolio $\mathrm{x}=\left(\mathrm{x}_{1}, \ldots, \mathrm{x}_{\mathrm{n}}\right)$ is finitely distributed over $\left\{\mathrm{R}_{\mathrm{x} 1}, \ldots, \mathrm{R}_{\mathrm{xT}}\right\}$, where $\mathrm{R}_{\mathrm{xi}}=\mathrm{x}_{1} \mathrm{r}_{\mathrm{i} 1}+\ldots+\mathrm{x}_{\mathrm{n}} \mathrm{r}_{\mathrm{in}}, \forall \mathrm{i} \in\{1, \ldots \mathrm{T}\}$.

\section{Mean-Risk Models}

\subsection{The general case}

Mean-risk models were developed in early fifties for the portfolio selection problem. In his seminal work "Portfolio selection”, Markowitz (1952) proposed variance as a risk measure. Since then, many alternative risk measures have been proposed. The question of which risk measure is most appropriate is still the subject of much debate.

In mean- risk models, two scalars are attached to each random variable: the expected value (mean) and the value of a risk measure. Preference is then defined using a trade-off between the mean where a larger value is desirable and risk where a smaller value is desirable:

In the mean-risk approach with the risk measure denoted by $\rho$, random variable $R_{x}$ dominates (is preferred to) random variable $R_{y}$ if and only if: $E\left(R_{x}\right) \geq E\left(R_{y}\right)$ and $\rho\left(R_{x}\right) \leq \rho\left(R_{y}\right)$ with at least one strict inequality. Alternatively, we can say that portfolio $\mathrm{x}$ dominates portfolio $\mathrm{y}$.

In this approach, the choice $\mathrm{x}$ (or the random variable $\mathrm{R}_{\mathrm{x}}$ ) is efficient (nondominated) if and only if there is no other choice y such that $\mathrm{R}_{\mathrm{y}}$ has higher expected value and less risk than $R_{x}$. This means that, for a given level of minimum expected return, $\mathrm{R}_{\mathrm{X}}$ has the lowest possible risk, and, for a given level of risk, it has the highest possible expected return. Plotting the efficient portfolios in a mean-risk space gives the efficient frontier.

Thus, the efficient solutions in a mean-risk model are Pareto efficient solutions of a multi-objective problem, in which the expected return is maximised and the risk is minimised:

$\operatorname{Max}\left\{\left(\mathrm{E}\left(\mathrm{R}_{\mathrm{x}}\right),-\rho\left(\mathrm{R}_{\mathrm{x}}\right)\right): \mathrm{x} \in \mathcal{A}\right\}$

Generally, for a multi-objective problem:

$\operatorname{Max}\left\{\mathrm{f}(\mathrm{x})=\left(\mathrm{f}_{1}(\mathrm{x}), \ldots, \mathrm{f}_{\mathrm{T}}(\mathrm{x})\right): \mathrm{x} \in \mathcal{A}\right\}$,

the Pareto preference relation is defined as follows:

A feasible solution $\mathrm{x}^{1} \in \mathcal{A}$ Pareto dominates another feasible solution $\mathrm{x}^{2} \in \mathcal{A}$ if $\mathrm{f}_{\mathrm{i}}\left(\mathrm{x}^{1}\right) \geq \mathrm{f}_{\mathrm{i}}\left(\mathrm{x}^{2}\right)$ for all $\mathrm{i}$ with at least one strict inequality. 
$\mathrm{x}_{0}$ is a Pareto efficient (non-dominated) solution of (2) if and only if there does not exist a feasible $\mathrm{x}$ such that $\mathrm{x}$ Pareto dominates $\mathrm{x}_{0}$. In other words, a Pareto efficient solution is a feasible solution such that, in order to improve upon one objective function, at least one other objective function must assume a worse value.

In order to find an efficient portfolio, we solve an optimisation problem with decision variables $\mathrm{x}_{1}, \ldots \mathrm{x}_{\mathrm{n}}$ :

Minimise $\rho\left(R_{x}\right)$

Subject to: $\mathrm{E}\left(\mathrm{R}_{\mathrm{x}}\right) \geq \mathrm{d}$ and $\left(\mathrm{x}_{1}, \ldots \mathrm{x}_{\mathrm{n}}\right\} \in \mathcal{A}$,

where d represents the desired level of expected return for the portfolio.

Varying $\mathrm{d}$ and repeatedly solving the corresponding optimisation problem identifies the minimum risk portfolio for each value of $d$. These are the efficient portfolios that compose the efficient set. By plotting the corresponding values of the objective function and of the expected return respectively in a return- risk space, we trace out the efficient frontier.

An alternative formulation, which explicitly trades risk against return in the objective function, is

Maximize $\quad E\left(R_{X}\right)-\tau \rho\left(R_{X}\right) \quad(\tau \geq 0)$

Subject to: $\left(\mathrm{x}_{1}, \ldots \mathrm{x}_{\mathrm{n}}\right\} \in \mathcal{A}$.

Varying the trade-off coefficient $\tau$ and repeatedly solving the corresponding optimisation problems traces out the efficient frontier.

\subsection{The mean-variance model}

The variance of a random variable $\mathrm{R}_{\mathrm{x}}$ is defined as its second central moment: $\sigma^{2}\left(R_{x}\right)=E\left[\left(R_{x}-E\left(R_{X}\right)\right)^{2}\right]$.

An important property is that the variance of the portfolio return $\mathrm{R}_{\mathrm{x}}=\mathrm{x}_{1} \mathrm{R}_{1}+\ldots+\mathrm{x}_{\mathrm{n}} \mathrm{R}_{\mathrm{n}}$, resulting from choice $\left(\mathrm{x}_{1}, \ldots, \mathrm{x}_{\mathrm{n}}\right)$, can be expressed as:

$\sigma^{2}\left(R_{x}\right)=\sum_{k=1}^{n} \sum_{j=1}^{n} x_{k} x_{j} \sigma_{k j}$, where $\sigma_{\mathrm{kj}}$ is the covariance of $\mathrm{R}_{\mathrm{k}}$ and $\mathrm{R}_{\mathrm{j}}$,

and thus variance is expressed as a quadratic function of $\mathrm{x}_{1}, \ldots, \mathrm{x}_{\mathrm{n}}$.

The mean-variance model can be formulated for the portfolio selection problem as follows:

Minimize $\sum_{k=1}^{n} \sum_{j=1}^{n} x_{k} x_{j} \sigma_{k j}$

Subject to

$$
\begin{aligned}
& \sum_{j=1}^{n} \mu_{j} x_{j} \geq d \\
& \sum_{i=1}^{n} x_{j}=1 \\
& x_{j} \geq 0, \forall j=1 . . n
\end{aligned}
$$


where $\mu_{\mathrm{j}}=$ the expected rate of return of asset $\mathrm{j}, \mathrm{j} \in\{1, \ldots, \mathrm{n}\}$;

$\sigma_{\mathrm{kj}}=$ the covariance between returns of asset $k$ and asset $j$, with $k, j \in\{1, \ldots, n\}$; $\mathrm{d}=$ the desired expected value of the portfolio return.

\subsection{The mean-CVaR model}

Let $\mathrm{R}_{\mathrm{x}}$ be a random variable representing the return of a portfolio $\mathrm{x}$ over a given holding period and $A \%=\alpha \in(0,1)$ a percentage which represents a sample of "worst cases" for the outcomes of $\mathrm{R}_{\mathrm{x}}$ (usually, $\alpha=0.01$ or $\alpha=0.05$ ).

The definition of CVaR at the specified level $\alpha$ is the mathematical transcription of the concept "average of losses in the worst A\% of cases" (Acerbi and Tasche 2002), where a "loss" is a negative outcome of $R_{x}$ (thus the loss associated with $R_{x}$ is described by the random variable $-\mathrm{R}_{\mathrm{x}}$ ).

Formally, the Conditional Value-at-Risk at level $\alpha$ of $\mathrm{R}_{\mathrm{X}}$ is defined as minus the mean of the $\alpha$-tail distribution of $R_{x}$, where the $\alpha$-tail distribution is obtained by taking the lower $\alpha$ part of the distribution of $\mathrm{R}_{\mathrm{x}}$ (corresponding to extreme unfavourable outcomes) and rescaling its distribution function to span $[0,1]$ :

$\operatorname{CVaR}_{\alpha}\left(R_{x}\right)=-\frac{1}{\alpha}\left\{E\left(R_{X} 1_{\left\{R_{X} \leq q^{\alpha}\left(R_{x}\right)\right\}}\right)-q^{\alpha}\left(R_{x}\right)\left[P\left(R_{X} \leq q^{\alpha}\left(R_{x}\right)\right)-\alpha\right]\right\}$

where $\mathrm{q}^{\alpha}$ is an $\alpha$-quantile of $\mathrm{R}_{\mathrm{x}}$, meaning that $\mathrm{P}\left(\mathrm{R}_{\mathrm{x}}<\mathrm{r}\right) \leq \alpha \leq \mathrm{P}\left(\mathrm{R}_{\mathrm{x}} \leq \mathrm{r}\right)$ (see Laurent 2003 for more details on $\alpha$-quantiles),

and $1_{\text {\{Relation\} }}=1$, if Relation is true

0 , if Relation is false.

(see Rockafellar and Uryasev 2000, 2002 for more details).

An important result, proved by Rockafellar and Uryasev (2000, 2002), and independently by Ogryczak and Ruszczynski (2002), is that the CVaR of a random variable $\mathrm{R}_{\mathrm{x}}$ can be calculated by solving a convex optimisation problem. Moreover, $\mathrm{CVaR}$ can be minimised over the set of feasible decision vectors. These results are summarised below:

Proposition 3.1 (CVaR calculation and optimisation): Let $\mathrm{R}_{\mathrm{x}}$ be a random variable depending on a decision vector $\mathrm{x}$ that belongs to a feasible set $\mathcal{A}$, and $\alpha \in(0,1)$. Consider the function:

$F_{\alpha}(x, v)=\frac{1}{\alpha} E\left\{\left[-R_{x}+v\right]^{+}\right\}-v$, where

$[\mathrm{u}]^{+}=\mathrm{u}$ for $\mathrm{u} \geq 0$

$[\mathrm{u}]^{+}=0$ for $\mathrm{u}<0$.

Then:

(a) As a function of $\mathrm{v}, F_{\alpha}$ is finite and continuous (hence convex) and $C \operatorname{VaR}_{\alpha}\left(R_{x}\right)=\min _{v \in R} F_{\alpha}(x, v)$.

\footnotetext{
${ }^{2}$ This is not necessarily the same as "the expected value of losses exceeding VaR at confidence level $\alpha$ ", as it is defined in earlier papers on CVaR. The two definitions lead to the same results when the distribution of the random variable under consideration is continuous, but differences may appear when the considered distribution has discontinuities -see Acerbi and Tasche 2002, Rockafellar and Uryasev 2002 for more details.
} 
In addition, the set consisting of the values of $\mathrm{v}$ for which the minimum is attained, denoted by $\mathrm{A}_{\alpha}(\mathrm{x})$, is a non-empty, closed and bounded interval (possibly formed by just one point).

(b) Minimising $C V a R_{\alpha}$ with respect to $\mathrm{x} \in \mathcal{A}$ is equivalent to minimising $F_{\alpha}$ with respect to $(\mathrm{x}, \mathrm{v}) \in \mathcal{A x R}$ :

$\min _{x \in A} \operatorname{CVaR}_{\alpha}\left(R_{x}\right)=\min _{(x, v) \in A x R} F_{\alpha}(x, v)$.

In addition, a pair $\left(\mathrm{x}^{*}, \mathrm{v}^{*}\right)$ minimises the right hand side if and only if $\mathrm{x}^{*}$ minimises the left hand side and $\mathrm{v}^{*} \in \mathrm{A}_{\alpha}\left(\mathrm{x}^{*}\right)$.

(c) $C \operatorname{VaR} R_{\alpha}\left(R_{x}\right)$ is convex with respect to $\mathrm{x}$ and $F_{\alpha}(x, v)$ is convex with respect to $(\mathrm{x}, \mathrm{v})$.

Thus, if the set $\mathcal{A}$ of feasible decision vectors is convex (which is the case for the basic version of the portfolio selection problem), and even if we impose a further lower limit on the expected return, minimising $\mathrm{CVaR}$ is a convex optimisation problem.

In the case when $R_{x}$ is a discrete random variable (as described in Section 2), calculating and optimising $\mathrm{CVaR}$ are linear programming problems. Suppose that $\mathrm{R}_{\mathrm{x}}$ has $\mathrm{T}$ possible outcomes $\mathrm{R}_{\mathrm{x} 1}, \ldots, \mathrm{R}_{\mathrm{xT}}$ with probabilities $\mathrm{p}_{1}, \ldots, \mathrm{p}_{\mathrm{T}}$. Then:

$F_{\alpha}(x, v)=\frac{1}{\alpha} \sum_{i=1}^{T} p_{i}\left[v-R_{x i}\right]^{+}-v$.

For the portfolio selection problem, as presented in Section 2, where $\mathrm{R}_{\mathrm{xi}}=\sum_{j=1}^{n} x_{j} r_{i j}$ with $r_{i j}$ the return of asset $j$ under scenario $i$,

$F_{\alpha}(x, v)=\frac{1}{\alpha} \sum_{i=1}^{T} p_{i}\left[v-\sum_{j=1}^{n} x_{j} r_{i j}\right]^{+}-v$.

Thus, the mean-CVaR $\mathrm{R}_{\alpha}$ model can be formulated for the portfolio selection problem as follows:

Minimise $-v+\frac{1}{\alpha} \sum_{i=1}^{T} p_{i} y_{i}$

Subject to

$$
\begin{aligned}
& \sum_{j=1}^{n}-r_{i j} x_{j}+v \leq y_{i}, \forall i=1, . ., T \\
& y_{i} \geq 0, \forall i=1, . ., T \\
& \sum_{j=1}^{n} \mu_{j} x_{j} \geq d \\
& \sum_{j=1}^{n} x_{j}=1 \\
& x_{j} \geq 0, \forall j=1, . ., n
\end{aligned}
$$




\section{The Mean-Variance-CVaR model}

\subsection{The theoretical background}

In this section, a model for portfolio selection is proposed, in which random variables are described by three statistics: the expected value, the variance and the $\mathrm{CVaR}$ at a specified confidence level $\alpha \in(0,1)$. We claim that taking three parameters into consideration, instead of two, gives a better modelling power. The proposed model may bring an improvement in the solution, in the case where a mean-variance efficient portfolio has an excessively large $\mathrm{CVaR}$, or a mean-CVaR efficient portfolio has an excessively large variance.

The idea of restricting the risk of a distribution from two different perspectives has been used before in various contexts.

Konno et al. (1993) proposed a "mean- absolute deviation skewness portfolio optimisation model", in which the lower semi-third moment of the portfolio return is maximised subject to constraints on the mean and on the absolute deviation of the portfolio return. A “mean- variance-skewness portfolio optimisation model” was proposed by Konno et al. (1995): they maximised the third moment of the portfolio return subject to constraints on the mean and on the variance of the portfolio return. Optimisation approaches are provided, in which the corresponding cubic and quadratic functions are approximated by linear functions.

Wang (2000) proposed a model in which the portfolio return has constraints on both variance and Value-at-Risk (VaR), and a maximum expected return under these conditions. However, no practical optimisation approach is provided.

Harvey et al. (2003) proposed a model in which random variables are chosen with respect to their expected value, variance and skewness. Thus, it may be considered that they use two risk measures in order to control the selection of a solution: the variance and the negative of skewness. Their model has a distributional assumption for portfolio returns and uses an expected utility maximisation approach, with the utility function depending on the expected value, variance and skewness.

Jorion (2003) proposed that a portfolio return distribution should have constraints on both variance and "tracking error volatility", which is "the volatility of the deviation of the active portfolio from the benchmark", with a maximum expected return under these conditions. Thus, this approach may also fall into the category of index-tracking models.

There have been various formulations of portfolio selection problems as multiple criteria models (see for example Ogryczak 2000, 2002). However, to the best of our knowledge, the use of CVaR together with variance within a multi-attribute model is novel. A categorised bibliography on the applications of multiple criteria decisionmaking techniques in finance is provided in Steuer and Na (2003).

The model proposed in this paper does not assume a particular distribution for the returns and, in addition, is convenient from a computational point of view. We define a preference relation for random variables and provide an optimisation approach for finding the efficient solutions with respect to this preference relation.

Consider again the portfolio selection problem described in Section 2, with the random variable $\mathrm{R}_{\mathrm{x}}$ and $\mathrm{R}_{\mathrm{y}}$ describing the returns of portfolios $\mathrm{x}$ and $\mathrm{y}$ respectively, with $\mathrm{x}, \mathrm{y} \in \mathcal{A}$. 
We consider a model for choice under risk that we refer to as the mean-varianceCVaR model, in which the preference relation among random variables is defined as follows:

In the mean-variance-CVaR model, a random variable $\mathrm{R}_{\mathrm{x}}$ is preferred to a random variable $\mathrm{R}_{\mathrm{y}}$ (or, similarly, the portfolio $\mathrm{x}$ is preferred to portfolio $\mathrm{y}$ ) if and only if $\mathrm{E}\left(\mathrm{R}_{\mathrm{x}}\right) \geq \mathrm{E}\left(\mathrm{R}_{\mathrm{y}}\right), \sigma^{2}\left(R_{x}\right) \leq \sigma^{2}\left(R_{y}\right)$ and $\mathrm{CVaR}_{\alpha}\left(\mathrm{R}_{\mathrm{x}}\right) \leq \mathrm{CVaR}_{\alpha}\left(\mathrm{R}_{\mathrm{y}}\right)$, with at least one strict inequality.

Thus, the non-dominated (efficient) solutions in the mean-variance-CVaR model are the Pareto efficient solutions of a multi-objective problem in which the expected value is maximised while the variance and the $\mathrm{CVaR}$ are minimised:

(MVC): $\max \left(\mathrm{E}\left(\mathrm{R}_{\mathrm{x}}\right),-\sigma^{2}\left(R_{x}\right),-\mathrm{CVaR}_{\alpha}\left(\mathrm{R}_{\mathrm{x}}\right)\right)$

Subject to: $\mathrm{x} \in \mathcal{A}$.

When plotting the efficient solutions in a mean-variance-CVaR space, a surface is obtained; we refer to this surface as "the efficient frontier" of the meanvariance-CVaR model.

\subsection{An optimisation approach}

The next issue to address is how to obtain the efficient solutions of the meanvariance-CVaR model.

Firstly, the multi-objective problem (MVC) is transformed into a single objective problem in which one objective function is optimised while lower limits are imposed on the remaining objective functions and transformed into constraints. This method, known in multi-objective optimisation as the " $\varepsilon$-constraint method" (Haimes et al. 1971, see also Steuer 1986) generally requires some regularization in order to guarantee that an optimal solution of the single-objective problem obtained is a Pareto optimal solution of the original multi-objective problem.

We choose to minimise variance for two reasons. Firstly, it is more intuitively appealing to impose limits on the expected value and $\mathrm{CVaR}$, rather than on variance. Secondly, we show that minimising variance is more convenient from a computational point of view. In either case, a convex optimisation problem would be obtained $^{3}$, irrespective of which statistic we choose for the objective function, but, when optimising variance, a quadratic programming problem is obtained, as shown below.

In what follows, for a random variable $\mathrm{R}_{\mathrm{x}}$ that depends on the decision vector $\mathrm{x}$, the variance of $\mathrm{R}_{\mathrm{x}}$ is denoted alternatively by $\sigma^{2}(x)$ or $\sigma^{2}\left(R_{x}\right)$. Similarly, the Conditional Value-at-Risk at level $\alpha$ of $\mathrm{R}_{\mathrm{x}}$ is denoted by $\mathrm{CVaR}_{\alpha}(\mathrm{x})$ or $\mathrm{CVaR}_{\alpha}\left(\mathrm{R}_{\mathrm{x}}\right)$, and the expected value of $\mathrm{R}_{\mathrm{x}}$ by $\mathrm{E}(\mathrm{x})$ or $\mathrm{E}\left(\mathrm{R}_{\mathrm{x}}\right)$.

We consider the following optimisation problem:

(P1): $\min \sigma^{2}(x)$

\footnotetext{
${ }^{3}$ As stated in Proposition 3.1, CVaR is a convex function of $\mathrm{x}$. Variance is also convex of $\mathrm{x}$, since the variance-covariance matrix is positive semi-definite. The expected value is linear thus convex of $\mathrm{x}$.
} 
Subject to: $\mathrm{CVaR}_{\alpha}(\mathrm{x}) \leq \mathrm{z}$

$$
\begin{gathered}
\mathrm{E}(\mathrm{x}) \geq \mathrm{d} \\
\mathrm{x} \in \mathcal{A} .
\end{gathered}
$$

where $\mathrm{z}$ and $\mathrm{d}$ are real numbers.

It is easy to prove that: if $\mathrm{x}^{*}$ is a Pareto optimal solution of (MVC) then $\mathrm{x}^{*}$ is also an optimal solution of (P1) with $\mathrm{z}=\mathrm{CVaR}_{\alpha}\left(\mathrm{x}^{*}\right)$ and $\mathrm{d}=\mathrm{E}\left(\mathrm{x}^{*}\right)$.

Indeed, assume that $\mathrm{x}^{*}$ is not an optimal solution of (P1). Obviously $\mathrm{x}^{*}$ is a feasible solution of (P1). Denote by $\mathrm{x}^{\prime}$ an optimal solution of (P1). It follows that $\sigma^{2}\left(x^{\prime}\right)<\sigma^{2}\left(x^{*}\right), \operatorname{CVaR}_{\alpha}\left(x^{\prime}\right) \leq \operatorname{CVaR}_{\alpha}\left(x^{*}\right)$ and $E\left(x^{\prime}\right) \geq E\left(x^{*}\right)$, which means that $x^{\prime}$ Pareto dominates $\mathrm{x}^{*}$ and we have a contradiction.

The converse is also true, with the additional assumption of uniqueness of the optimal solution:

If $\mathrm{x}^{*}$ is the unique optimal solution of (P1), then $\mathrm{x}^{*}$ is also a Pareto optimal solution of (MVC).

Indeed, assume that $\mathrm{x}^{*}$ is Pareto dominated in (MVC) and denote by $\mathrm{x}^{\prime}$ a point that Pareto dominates $x^{*}$. This means that $\sigma^{2}\left(x^{\prime}\right) \leq \sigma^{2}\left(x^{*}\right), \operatorname{CVaR}_{\alpha}\left(x^{\prime}\right) \leq \operatorname{CVaR}_{\alpha}\left(x^{*}\right) \leq \mathrm{z}$ and $\mathrm{E}\left(\mathrm{x}^{\prime}\right) \geq \mathrm{E}\left(\mathrm{x}^{*}\right) \geq \mathrm{d}$ with at least one strict inequality. Thus $\mathrm{x}^{\prime}$ is another feasible solution of (P1) such that $\sigma^{2}\left(\mathrm{x}^{\prime}\right) \leq \sigma^{2}\left(\mathrm{x}^{*}\right)$, which is a contradiction.

Remark 4.1: If the covariance matrix of returns is positive definite, then variance is a strictly convex function of $\mathrm{x}$. In this case, minimising variance over a convex set has at most one optimal solution; thus, the possibility of multiple optimal solutions for (P1) is eliminated. This is usually the case; if there are no redundant assets (ones that can be replicated by the remaining of the assets) or risk-free assets in the collection of assets considered, then the covariance matrix is positive definite.

We summarise these results below:

Proposition 4.1: If the covariance matrix is positive definite, a point $\mathrm{x}^{*}$ is a Pareto efficient solution of (MVC) if and only if $x^{*}$ is an optimal solution of (P1) with $\mathrm{z}=\mathrm{CVaR}_{\alpha}\left(\mathrm{x}^{*}\right)$ and $\mathrm{d}=\mathrm{E}\left(\mathrm{x}^{*}\right)$.

Thus, in the case of a positive definite covariance matrix of returns, the Pareto efficient solutions of (MVC) can be fully characterised as optimal solutions in (P1) with active constraints on mean and on CVaR.

In fact, the above statement is true for the general case of a (positive semidefinite) covariance matrix - see Proposition A1 in Appendix A. Also, in Appendix A we present a method for obtaining all the Pareto efficient solutions of (MVC) for the general case when the covariance matrix of returns is positive semi-definite.

The next issue that arises is how to represent the CVaR constraint in (P1). As presented in Proposition 3.1, the function $F_{\alpha}(x, v)=\frac{1}{\alpha} E\left\{\left[v-R_{x}\right]^{+}\right\}-v$ may be used both for calculating the $\mathrm{CVaR}$ of a given random variable and for optimising $\mathrm{CVaR}$ with respect to all feasible decisions vectors.

Furthermore, Krokhmal et al. (2002) proved that the same function $F_{\alpha}(x, v)$ may be used for imposing an upper limit on the $\mathrm{CVaR}$ of a random variable, while maximising its expected value. 
Their result may be extended to a much more general case. In fact, the constraint " $\mathrm{CVaR}_{\alpha}(\mathrm{x}) \leq \mathrm{z}$ " can be replaced with the constraint " $F_{\alpha}(x, v) \leq \mathrm{z}$ ” in all optimisation problems, irrespective of the form of the objective function or the feasible set.

Proposition 4.2: Consider two optimisation problems (P) and (P') with $\mathcal{A} \subset \mathrm{R}^{\mathrm{n}}$ a feasible set of decision vectors and the objective function $\mathrm{f}: \mathrm{R}^{\mathrm{n}} \rightarrow \mathrm{R}$ of any form:

(P): $\min \mathrm{f}(\mathrm{x})$

Subject to: $\operatorname{CVaR}_{\alpha}(x) \leq \mathrm{z}$

$$
\mathrm{x} \in \mathcal{A}
$$

(P’): $\min \mathrm{f}(\mathrm{x})$

Subject to: $F_{\alpha}(x, v) \leq z$

$$
\mathrm{x} \in \mathcal{A}, \mathrm{v} \in \mathrm{R} \text {. }
$$

In (P), the variables are $\mathrm{x}_{1}, \ldots, \mathrm{x}_{\mathrm{n}}$ while in (P'), the variables are $\mathrm{x}_{1}, \ldots, \mathrm{x}_{\mathrm{n}}$ and $\mathrm{v}$.

Then: $(\mathrm{P})$ and ( $\left.\mathrm{P}^{\prime}\right)$ achieve the same optimal value. Moreover, a point $\mathrm{x}^{*} \in \mathrm{P}$ is an optimal solution for $(\mathrm{P})$ iff there exists $\mathrm{v}^{*} \in \mathrm{R}$ such that $\left(\mathrm{x}^{*}, \mathrm{v}^{*}\right)$ is an optimal solution for (P'). If, in addition, the constraint $C V a R_{\alpha}(x) \leq z$ in $(\mathrm{P})$ is active, then $\mathrm{v}^{*} \in \mathrm{A}_{\alpha}\left(\mathrm{x}^{*}\right)$ (meaning that $\left.F\left(x^{*}, v^{*}\right)=\min _{v \in R} F_{\alpha}\left(x^{*}, v\right)\right)$.

Proof: As stated in Proposition 3.1, $C V a R_{\alpha}(x)=\min _{v \in R} F_{\alpha}(x, v)$. Thus, the problem $(\mathrm{P})$ may be written as:

(P): $\min \mathrm{f}(\mathrm{x})$

$$
\begin{gathered}
\text { Subject to: } \min _{v \in R} F_{\alpha}(x, v) \leq z \\
\mathrm{x} \in \mathcal{A}
\end{gathered}
$$

Suppose now that $\mathrm{x}^{*}$ is an optimal solution for $(\mathrm{P})$. Obviously $\left(\mathrm{x}^{*}, \mathrm{~V}^{*}\right)$ is a feasible solution for $\left(\mathrm{P}^{\prime}\right)$, where $\mathrm{v}^{*}$ is such that $F\left(x^{*}, v^{*}\right)=\min _{v \in R} F_{\alpha}\left(x^{*}, v\right)$. Assume that there exists ( $\left.x^{\prime}, v^{\prime}\right)$ another feasible solution for ( $\left.P^{\prime}\right)$ such that $f\left(x^{\prime}\right)<f\left(x^{*}\right)$. Since $F_{\alpha}\left(x^{\prime}, v^{\prime}\right) \leq z$ it follows that $\min _{v \in R} F_{\alpha}\left(x^{\prime}, v\right) \leq z$; thus, $\mathrm{x}^{\prime}$ is a feasible solution of (P1) which improves the objective function as compared to $\mathrm{x}^{*}$, which is a contradiction.

Similarly, in a straightforward way, the converse may be proven; the last part of the proposition is obvious.

Thus, we consider another optimisation problem, with variables $\mathrm{x}=\left(\mathrm{x}_{1}, \ldots, \mathrm{X}_{\mathrm{n}}\right) \in \mathrm{A} \subset \mathrm{R}^{\mathrm{n}}$ and $\mathrm{v} \in \mathrm{R}$ :

(P2): $\min \sigma^{2}(x)$

$$
\begin{aligned}
& \text { Subject to: } F_{\alpha}(x, v) \leq \mathrm{z} \\
& \mathrm{E}(\mathrm{x}) \geq \mathrm{d} \\
& \mathrm{x} \in \mathcal{A}, \mathrm{v} \in \mathrm{R}
\end{aligned}
$$

where $\mathcal{A}$ is the (convex) set of feasible decision vectors, as given, for example, by (1).

The result below follows from Propositions 4.1 and 4.2:

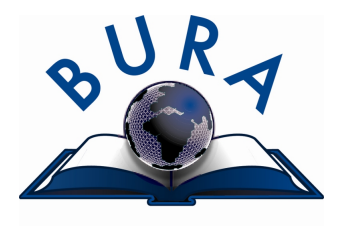


Proposition 4.3: If the covariance matrix of returns is positive definite, the Pareto efficient solutions of (MVC) are fully characterised as optimal solutions of (P2) with active constraints on mean and on $\mathrm{CVaR}^{4}$.

In other words, $x^{*}$ is a Pareto efficient solution of (MVC) if and only if there exists $\mathrm{v}^{*} \in \mathrm{R}$ such that $\left(\mathrm{x}^{*}, \mathrm{v}^{*}\right)$ is an optimal solution to (P2) with $z=F_{\alpha}\left(x^{*}, v^{*}\right)$ and $\mathrm{d}=\mathrm{E}\left(\mathrm{x}^{*}\right)$.

Therefore, varying $\mathrm{d}$ and $\mathrm{z}$ in the problem (P2) such that the constraints on $\mathrm{CVaR}$ and on the expected value are active produces all the efficient solutions of the mean-variance-CVaR model. As shown in Section 4.4, this means varying $\mathrm{d}$ and $\mathrm{z}$ between some finite limits that can be easily determined.

\subsection{Alternative optimisation approaches}

The optimisation approach described in the previous subsection is not unique. A commonly used method of obtaining a Pareto efficient solution of a multi-objective optimisation problem is to use a scalarizing function, meaning a real-valued function that is a composite of all objective functions. When optimised, the scalarizing function produces a Pareto efficient solution of the multi-objective optimisation problem. Thus, the problem is reduced to a single objective optimisation problem. We give below two examples of scalarizing functions, leading to two alternative optimisation approaches for the mean-variance-CVaR model.

The most common scalarizing function is a weighted sum of the objective functions in the original multi-objective optimisation problem. The general requirement on weights is that they should be strictly positive but usually they are normalised such they sum to 1 . In our case, the single objective optimisation problem that results is:

$\operatorname{Max} w_{1} E(x)-w_{2} \sigma^{2}(x)-w_{3} C V a R_{\alpha}(x)$

Subject to: $\mathrm{x} \in \mathcal{A}$

where $\mathrm{w}_{1}, \mathrm{w}_{2}, \mathrm{w}_{3}$ are strictly positive ${ }^{5}$.

It is clear that every optimal solution of (P3) is a Pareto efficient solution of (MVC).

The converse is not always true, in the sense that there may be Pareto optimal solutions of (MVC) that cannot be obtained as optimal solutions of a problem (P3) with strictly positive $\mathrm{w}_{1}, \mathrm{w}_{2}$ and $\mathrm{w}_{3}$ (for example, the Pareto optimal solution of (MVC) that globally minimises variance).

However, due to the convexity of all objective functions on (MVC), every Pareto optimal solution of (MVC) can be obtained as an optimal solution of (P3) with nonnegative weights (see Jahn 1985). For example, the Pareto optimal solution of (MVC) that globally minimises variance is obtained as an optimal solution of (P3) with $\mathrm{w}_{1}=$ $\mathrm{w}_{3}=0, \mathrm{w}_{2}=1$.

\footnotetext{
${ }^{4}$ This statement holds even without the assumption of a positive definite covariance matrix - the proof is given in Appendix A.

${ }^{5}$ If additionally there is the assumption of unique optimal solutions of (P3) when some of the weights are zero, then only the non-negativity condition is required for $\mathrm{w}_{1}, \mathrm{w}_{2}$ and $\mathrm{w}_{3}$.
} 
This approach has several disadvantages (see Das and Dennis 1997), one of them being the fact that the weights $\mathrm{w}_{1}, \mathrm{w}_{2}, \mathrm{w}_{3}$ are rather difficult to interpret. It is more meaningful to set desired levels of expected return and of CVaR and solve (P2).

Another example of a scalarizing function is obtained by considering target values (called reference points or aspiration points) for the values of the objective functions. This technique for multi-objective optimisation, named The Reference Point Method is fully described in Wierzbicki 1998. Consider the general multiobjective problem

(MO'): $\operatorname{Max}\left(\mathrm{f}_{1}(\mathrm{x}), \mathrm{f}_{2}(\mathrm{x}), \ldots, \mathrm{f}_{\mathrm{T}}(\mathrm{x})\right)$

Subject to: $\mathrm{x} \in \mathrm{X}$,

And let $\mathrm{w}^{*}{ }_{1}, \mathrm{w}^{*}, \ldots, \mathrm{w}^{*} \mathrm{~T}$ be the user-defined aspiration points for the objective functions. The simplest form of scalarizing function is:

$\gamma_{w^{*}}(x)=\min _{1 \leq k \leq T}\left(f_{k}(x)-w_{k}^{*}\right)+\varepsilon \sum_{k=1}^{T}\left(f_{k}(x)-w_{k}^{*}\right)$

where $\varepsilon>0$ is an arbitrary small parameter.

The terms $f_{k}(x)-w^{*}{ }_{k}$ in (4) are usually replaced by more complicated functions of $\mathrm{x}$ and $\mathrm{w}_{\mathrm{k}}, \gamma_{\mathrm{k}}\left(\mathrm{x}, \mathrm{w}_{\mathrm{k}}{ }_{\mathrm{k}}\right)$, which must satisfy certain properties (see for example Wierzbicki 1998, Makowski and Wierzbicki 2003). These functions are called partial achievement functions since they measure the actual achievement of the $\mathrm{k}$-th objective function with respect to its corresponding aspiration level $\mathrm{w}^{*} \mathrm{k}$. Various functions $\gamma_{\mathrm{k}}\left(\mathrm{x}, \mathrm{w}_{\mathrm{k}}^{*}\right)$ provide a wide modelling environment for measuring individual achievements. Other examples of such functions may be found in Wierzbicki 1998, Makowski and Wierzbicki 2003.

Provided that all the reference points lie between the lower and the upper bound of the corresponding objective function, the maximisation of (4) provides a Pareto efficient solution of (MO'). The converse is true, in the sense that for every Pareto efficient solution of (MO'), there exist aspiration levels such that this efficient solution maximises the corresponding achievement function (see Wierzbicki 1998).

In our case, the scalarizing achievement function to maximise is:

$$
\begin{aligned}
& \gamma_{w^{*}}(x)=\min \left\{E(x)-w^{*}, w^{*}{ }_{2}-\sigma^{2}(x), w^{*}{ }_{3}-\operatorname{CVaR}_{\alpha}(x)\right\}+ \\
&\left.+\varepsilon\left[E(x)-w^{*}{ }_{1}+w^{*}{ }_{2}-\sigma^{2}(x)+w^{*}{ }_{3}-C V a R_{\alpha}(x)\right\}\right]
\end{aligned}
$$

where $\varepsilon>0$ is an arbitrary small parameter.

The Reference Point Method is primarily designed for obtaining a specific solution of a multi-objective problem rather than the whole set of efficient solutions. Although all the efficient solutions may obtained with this method by choosing appropriate reference points, care must be taken in choosing the reference points between the lower and upper bound of each objective function. The lower bounds for the objective functions are difficult to find and often approximations are used.

In contrast, the optimisation method described in Section 4.2 produces the entire set of efficient solutions of the mean-variance-CVaR model with no difficulty, as described in the next section. 


\subsection{The efficient frontier of the mean-variance-CVaR model}

We consider the case when the covariance matrix of returns is positive definite; the general case of a positive semi-definite covariance matrix is treated in Appendix A.

As presented in Section 4.2, varying the right hand sides $\mathrm{d}$ and $\mathrm{z}$ in (P2) such that the corresponding constraints on mean and $\mathrm{CVaR}$ are active produces all the efficient solutions of (MVC).

Thus, the level $d$ for the expected value must lie in the interval $\left[\mathrm{d}_{\min }, \mathrm{d}_{\max }\right]$. We define $d_{\text {min }}=\max \left\{d_{\text {minvar }}, d_{\text {minCVaR }}\right\}$, where $d_{\text {minvar }}$ and $d_{\text {minCVaR }}$ are the expected returns of the minimum variance portfolio (mean-variance efficient) and minimum CVaR portfolio (mean-CVaR efficient) respectively. $\mathrm{d}_{\text {minvar }}$ may be found as the optimal value of the variable $\mathrm{d}_{0}$ in the problem:

$\min \sigma^{2}(x)$

$$
\begin{aligned}
\text { Subject to: } & E(x) \geq d_{0} \\
& x \in \mathcal{A}, d_{0} \in R .
\end{aligned}
$$

$d_{\text {minCVaR }}$ may be found as the optimal value of the variable $d_{1}$ in the problem:

$\min \mathrm{F}_{\alpha}(\mathrm{x}, \mathrm{v})$

$$
\begin{aligned}
& \text { Subject to: } \begin{aligned}
& E(x) \geq d_{1} \\
& x \in \mathcal{A}, v \in R, d_{1} \in R .
\end{aligned}
\end{aligned}
$$

To be more precise, $d_{\text {minCVaR }}$ may be found as above only when the minimisation of $\mathrm{F}_{\alpha}(\mathrm{x}, \mathrm{v})$ with respect to $(\mathrm{x}, \mathrm{v})$ over $\mathcal{A x R}$ provides a unique optimal solution. In the case of non-unique optimal solutions, we can obtain portfolios having the same minimal CVaR but different expected returns; among these, we are interested in the portfolio with the maximum expected return. To obtain this portfolio, we denote by $\mathrm{CVaR}_{\min }$ the optimal value of the above problem and solve another optimisation problem:

$\max \mathrm{E}(\mathrm{x})$

Subject to: $\mathrm{F}_{\alpha}(\mathrm{x}, \mathrm{v}) \leq \mathrm{CVaR} \min$ $\mathrm{x} \in \mathcal{A}, \mathrm{v} \in \mathrm{R}$.

We define $d_{\max }$ as the maximum possible expected return ${ }^{6}$ : the optimal value of the objective function in the problem:

$\max \mathrm{E}(\mathrm{x})$

Subject to: $\mathrm{x} \in \mathcal{A}$.

Furthermore, for a specific $d^{*} \in\left[d_{\min }, d_{\max }\right]$, the level $\mathrm{z}$ of $C \mathrm{CVR}_{\alpha}$ must lie in the interval $\left[\mathrm{z}_{\mathrm{d}^{*}, \min }, \mathrm{z}_{\mathrm{d}^{*}, \max }\right]$, where $\mathrm{z}_{\mathrm{d}^{*} \text {,min }}$ is the best (minimum) $C V a R_{\alpha}$ level for the expected return $\mathrm{d}^{*}$ and $\mathrm{z}_{\mathrm{d}^{*} \text {,max }}$ is the $C V a R_{\alpha}$ level of the (unique) portfolio that minimises variance for the expected return $\mathrm{d}^{*}$.

$\mathrm{z}_{\mathrm{d}^{*} \text {,min }}$ is the optimal value of the objective function in the problem:

\footnotetext{
${ }^{6} \mathrm{~d}_{\max }$ is also equal to the highest expected return of the component assets in the portfolio selection problem.
} 
Min $\mathrm{F}_{\alpha}(\mathrm{x}, \mathrm{v})$

Subject to: $\mathrm{E}(\mathrm{x}) \geq \mathrm{d}^{*}$

$$
\mathrm{x} \in \mathcal{A}, \mathrm{v} \in \mathrm{R} \text {. }
$$

$\mathrm{Z}_{\mathrm{d}^{*} \text {, max }}$ may be found as the optimal value of the objective function in the problem:

$\operatorname{Min} \mathrm{F}_{\alpha}\left(\mathrm{x}^{*}, \mathrm{v}\right)$

Subject to: $\mathrm{v} \in \mathrm{R}$,

where $\mathrm{x}^{*}=\left(\mathrm{x}^{*}{ }_{1}, \ldots, \mathrm{X}_{\mathrm{n}}\right)$ is the (unique) portfolio that minimises variance for the mean return $\mathrm{d}^{*}$.

The fact that the imposed limit $\mathrm{z}$ on $\mathrm{CVaR}_{\alpha}$ is greater than or equal to $\mathrm{z}_{\mathrm{d}^{*} \text {,min }}$ ensures that the problem (P2) is not infeasible, while $\mathrm{z}$ being less than or equal to $\mathrm{Z}_{\mathrm{d}^{*} \text {,max }}$ ensures that the constraint on CVaR in (P2) is active. When solving problem (P2) for a level of expected return equal to $\mathrm{d}^{*}$ and a $\mathrm{CVaR}$ level equal to $\mathrm{z}_{\mathrm{d}^{*} \text {,min }}$, we obtain a mean-CVaR efficient portfolio; more precisely, the mean-CVaR efficient portfolio with the lowest variance for expected return $\mathrm{d}^{*}$.

When solving problem (P2) for a level of expected return equal to $\mathrm{d}^{*}$ and a $\mathrm{CVaR}$ level equal to $\mathrm{z}_{\mathrm{d}^{*} \text {,max }}$, we obtain the mean-variance efficient portfolio with expected return $\mathrm{d}^{*}$.

For a fixed level of expected return, the efficient solutions in the meanvariance-CVaR model form a curve when plotted in a variance-CVaR space, where the lower end of this curve is represented by the mean- CVaR efficient solution (with the lowest variance) and the upper end is represented by the mean- variance efficient solution. The other points of this curve are not efficient in either the mean- variance or the mean-CVaR model.

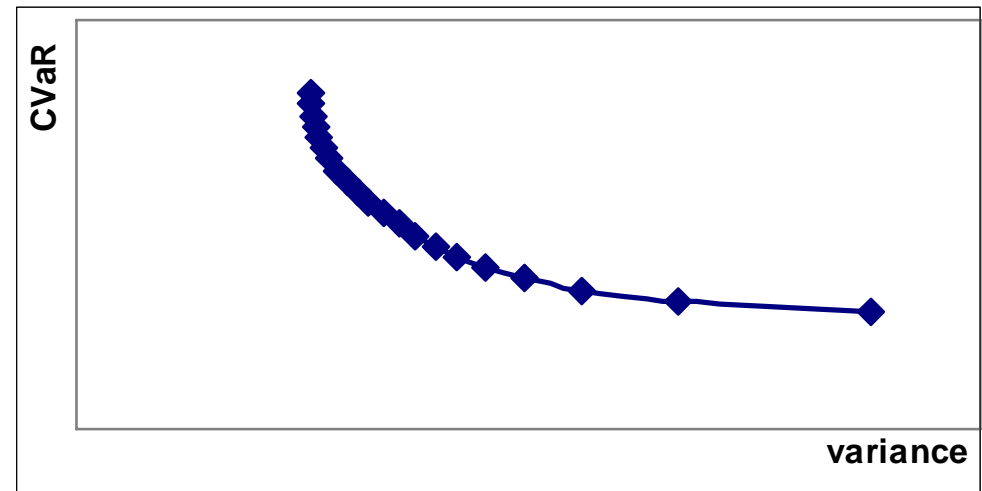

Fig. 4.1: The efficient solutions of the mean-variance-CVaR model, for a fixed level of expected value, plotted in a variance-CVaR space.

For the maximum level of expected return $d_{\max }$, this curve degenerates into just one point, with the coordinates equal to the variance and CVaR of the (only) efficient portfolio obtained for $\mathrm{d}_{\max }$, consisting of the asset with the highest expected return.

\subsection{The formulation of the mean-variance-CVaR model for scenario models}


For the portfolio selection problem, as presented in Section 2, consider $\mathrm{T}$ scenarios and $n$ assets with

$r_{i j}=$ the return of asset $j$ under scenario $i$, for $i=1 \ldots T$ and $j=1 \ldots n$;

$\mathrm{p}_{\mathrm{i}}=$ the probability of scenario $\mathrm{i}$ occurring, for $\mathrm{i}=1 \ldots \mathrm{T}$;

$\mu_{\mathrm{j}}=$ the expected return of asset $\mathrm{j}, \mathrm{j}=1 \ldots \mathrm{n}$;

$\sigma_{\mathrm{jk}}=$ the covariance between the returns of assets $\mathrm{j}$ and $\mathrm{k}$, for $\mathrm{j}, \mathrm{k}=1 \ldots \mathrm{n}$.

As presented in Section 3.3, the function $F_{\alpha}$ can be written as:

$$
F_{\alpha}(x, v)=\frac{1}{\alpha} \sum_{i=1}^{T} p_{i}\left[v-\sum_{j=1}^{n} x_{j} r_{i j}\right]^{+}-v .
$$

Thus, we write the mean-variance-CVaR model as:

$\operatorname{Min} \sum_{j, k=1}^{n} x_{j} x_{k} \sigma_{j k}$

Subject to:

$\sum_{j=1}^{n} x_{j} \mu_{j} \geq d$

$\frac{1}{\alpha} \sum_{i=1}^{T} p_{i} y_{i}-v \leq z$

$y_{i} \geq v-\sum_{j=1}^{n} x_{j} r_{i j}, \quad \forall \mathrm{i} \in\{1, \ldots, \mathrm{T}\}$

$y_{i} \geq 0, \forall \mathrm{i} \in\{1, \ldots, \mathrm{T}\}$

$\sum_{j=1}^{n} x_{j}=1$

$x_{j} \geq 0 \forall \mathrm{j} \in\{1, \ldots, \mathrm{n}\}$

The minimisation is over $\mathrm{v}, \mathrm{x}_{1}, \ldots, \mathrm{x}_{\mathrm{n}}, \mathrm{y}_{1}, \ldots, \mathrm{y}_{\mathrm{T}}$.

\section{Computational results}

\subsection{The data set and methodology}

The purpose of this section is to investigate the practical performance of the mean-variance-CVaR model as compared to that of the mean-variance or mean-CVaR model. Precisely, for several levels of expected return, we select portfolios that are efficient in the mean-variance-CVaR model, but dominated in the mean-variance or mean-CVaR model, and we also consider the corresponding mean-variance efficient portfolio and the mean-CVaR efficient portfolio. We compare their in-sample and out-of-sample performances.

We use CVaR at 0.01 confidence level.

A dataset, drawn from the FTSE 100 index, was used for this analysis. The returns of the 76 stocks that belonged to the index throughout the period January 1993December 2003 were considered (for each of the remaining 24 stocks data there is at least one missing data item in the specified period). The dataset consists of monthly returns and has 132 time periods, considered as equally probable scenarios $(n=76$, $\mathrm{T}=132$ ). For the out-of sample analysis, the behaviour of the portfolios obtained was 
examined over the eighteen months following the date of selection (January 2004June 2005). The models were written in the MPL modelling language (Maximal Software Inc. 2000) and processed using CPLEX 9.0 optimisation solver (ILOG 2003). The matrix of covariances of the returns is computed from historical data.

\subsection{In-sample analysis}

We consider six levels of expected return, which divide the interval $\left[\mathrm{d}_{\min }, \mathrm{d}_{\max }\right]$ (see Section 4.4) into 5 equal parts: $d_{1}=d_{\min }=0.009268, d_{2}=0.014034, d_{3}=0.018801$, $\mathrm{d}_{4}=0.023567, \mathrm{~d}_{5}=0.028334, \mathrm{~d}_{6}=\mathrm{d}_{\max }=0.0331$. For each level of expected return $\mathrm{d}_{\mathrm{i}}$, with $\mathrm{i}=1 . . .5$, we determine $\mathrm{z}_{\mathrm{di} \text {,min: }}$ the minimum level of CVaR (corresponding to the mean-CVaR efficient portfolio) and $\mathrm{z}_{\mathrm{di} \text {,max }}$ : the maximum level of $\mathrm{CVaR}$ (the lowest CVaR of a mean-variance efficient portfolio with expected return $d_{i}$ ) and, between them, another 3 equally spaced levels of $C V a R$. Thus, the interval $\left[z_{d i, m i n}, z_{d i, m a x}\right]$ for $\mathrm{CVaR}$ is divided into 4 equal parts. For a specific level of expected return, when solving the mean-variance-CVaR model with these CVaR levels, we obtain 5 portfolios, denoted by: $\mathrm{P}_{\mathrm{CVaR}}, \mathrm{P}_{1 / 4 \mathrm{CV} a R}, \mathrm{P}_{1 / 2 \mathrm{CVaR}}, \mathrm{P}_{3 / 4 \mathrm{CVaR}}$ and $\mathrm{P}_{\mathrm{var}}$ respectively. Thus, $\mathrm{P}_{\mathrm{CVaR}}$ is the mean-CVaR efficient portfolio (with the lowest variance, for the specified expected return) and $\mathrm{P}_{\mathrm{var}}$ is the (unique) mean-variance efficient portfolio for the specified expected return (see fig. 5.1) ${ }^{7}$.

We first investigate the composition of the considered portfolios. For all levels of expected return, the mean-variance efficient portfolios have considerably more assets in their composition than the mean-CVaR efficient portfolios. This was expected, since the "diversification effect" is the basis of the mean-variance theory.

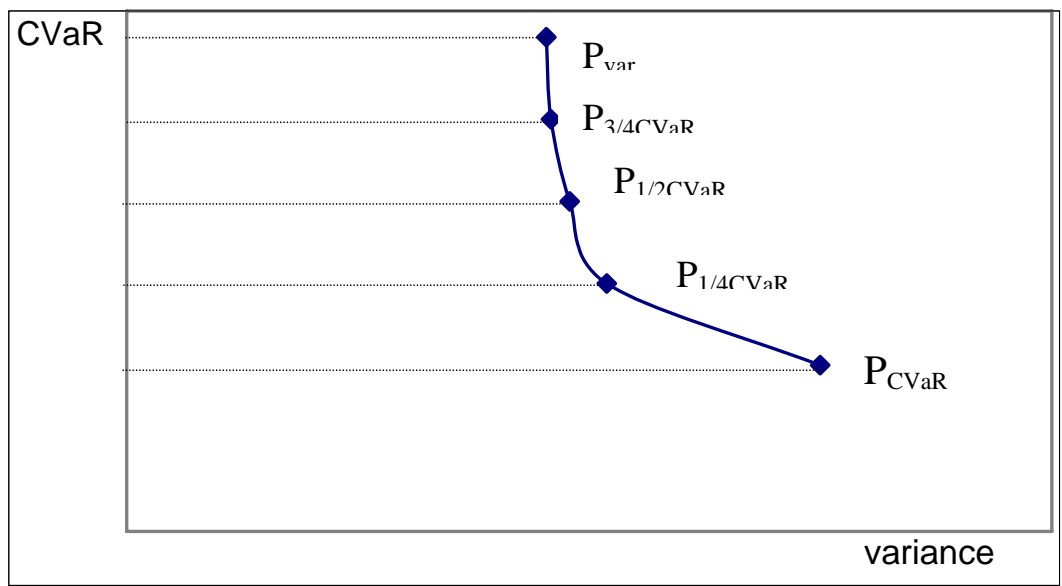

Fig. 5.1: The efficient frontier for a fixed level of expected return, in a variance-CVaR space. The interval for CVaR is divided into 4 equal parts.

The other three portfolios $\mathrm{P}_{1 / 4 C V a R}, \mathrm{P}_{1 / 2 \mathrm{CVaR}}, \mathrm{P}_{3 / 4 \mathrm{CVaR}}$ have usually a number of assets in composition significantly higher than mean-CVaR efficient portfolios, but usually smaller than mean-variance efficient portfolios. There are cases in which these portfolios are as well as or even more diversified than the mean-variance efficient portfolios (see Table 5.1 below); we notice that this happens when the expected return of the portfolio is high, thus, at high levels of risk. However, in most cases, the

\footnotetext{
${ }^{7}$ The CVaR level of $\mathrm{P}_{1 / 2 \mathrm{CVaR}}$ is the arithmetic mean of the CVaR levels of $\mathrm{P}_{\mathrm{CVaR}}$ and $\mathrm{P}_{\mathrm{var}}$. Similarly, the CVaR level of $\mathrm{P}_{1 / 4 \mathrm{CVaR}}$ is the arithmetic mean of the CVaR levels of $\mathrm{P}_{\mathrm{CVaR}}$ and $\mathrm{P}_{1 / 2 \mathrm{CVaR}}$.
} 
number of assets in the composition increases while the level of variance decreases (and the level of CVaR increases). Generally, the assets there are in the composition of mean-CVaR efficient portfolios are also in the composition of portfolios with a higher CVaR level. However, there are assets in the composition of the mean-CVaR portfolios but not in the composition of portfolios with a higher CVaR level. This aspect happens for small portfolio expected returns, thus, at low levels of risk. It may be noticed that, while the expected portfolio return (and thus the risk) increases, those assets are no longer in the composition of any efficient portfolio.

The portfolio weights of the efficient portfolios considered are presented in Appendix B.

\begin{tabular}{|l|c|c|c|c|c|}
\hline & $\mathbf{P}_{\text {CVaR }}$ & $\mathbf{P}_{1 / 4 \mathrm{CVaR}}$ & $\mathbf{P}_{1 / 2 \mathrm{CVaR}}$ & $\mathbf{P}_{3 / 4 \mathrm{CVaR}}$ & $\mathbf{P}_{\mathrm{var}}$ \\
\hline $\mathrm{d}_{1}=0.00927$ & 10 & 17 & 20 & 22 & 23 \\
\hline $\mathrm{d}_{2}=0.01403$ & 12 & 16 & 20 & 21 & 21 \\
\hline $\mathrm{d}_{3}=0.01880$ & 8 & 11 & 13 & 12 & 13 \\
\hline $\mathrm{d}_{4}=0.02357$ & 5 & 7 & 8 & 8 & 7 \\
\hline $\mathrm{d}_{5}=0.02833$ & 3 & 4 & 5 & 6 & 6 \\
\hline
\end{tabular}

Table 5.1: The number of assets in the composition of mean-variance-CVaR efficient portfolios.

We next investigate the in-sample performances of $\mathrm{P}_{1 / 4 C V a R}, \mathrm{P}_{1 / 2 \mathrm{CVaR}}, \mathrm{P}_{3 / 4 C V a R}$, as compared with those of $\mathrm{P}_{\mathrm{CVaR}}$ and $\mathrm{P}_{\mathrm{var}}$. We analyse their return distributions using common in sample parameters. Obviously, the CVaR levels of $\mathrm{P}_{1 / 4 \mathrm{CVaR}}, \mathrm{P}_{1 / 2 \mathrm{CVaR}}$, $\mathrm{P}_{3 / 4 C V a R}$ are better than the $\mathrm{CVaR}$ of $\mathrm{P}_{\mathrm{var}}$. On the other hand, their variance is generally significantly smaller than that of $\mathrm{P}_{\mathrm{CVaR}}$. All the other in-sample parameters are between those of $\mathrm{P}_{\mathrm{CVaR}}$ and $\mathrm{P}_{\mathrm{var}}$. In most cases, $\mathrm{P}_{\mathrm{CVaR}}$ has the return distribution with the best skewness, kurtosis and minimum of returns but also with the worst variance. In contrast, $\mathrm{P}_{\mathrm{var}}$ has the return distribution with the best variance but usually the worst skewness, kurtosis and minimum of returns. This is in line with the modelling paradigm since minimisation of CVaR leads to reduction in the (weighted) tail of the resulting portfolio return distribution. The other portfolios $\mathrm{P}_{1 / 4 C V a R}, \mathrm{P}_{1 / 2 \mathrm{CVaR}}, \mathrm{P}_{3 / 4 C V a R}$ represent a compromise in between these two "extremes". Their return distribution improves in the left tail, as compared with $\mathrm{P}_{\mathrm{var}}$ and also has a significantly smaller spread around the mean, as compared with $\mathrm{P}_{\mathrm{CVaR}}$. In particular, $\mathrm{P}_{1 / 4 C V a R}$ has return distributions with the variance significantly smaller than that of $\mathrm{P}_{\mathrm{CVaR}}$ at the expense of a relatively small increase in CVaR. This aspect can be seen from Tables 5- 10 (with the best values in italic bold and the worst values enclosed by rectangles) in Appendix C and is also illustrated in Fig. 5.1.

In Fig. 5.2 below the histogram of the return distribution of $\mathrm{P}_{\mathrm{CVaR}}$ for expected return $\mathrm{d}_{1}=0.00927$ is presented. This distribution is positively skewed, with a short left tail, a long right tail and a large probability of outcomes below the expected value. Therefore, the probability of large losses is very small, but there is a large probability of small losses. In addition, this distribution is particularly "flat", that is, not concentrated around the expected value. 


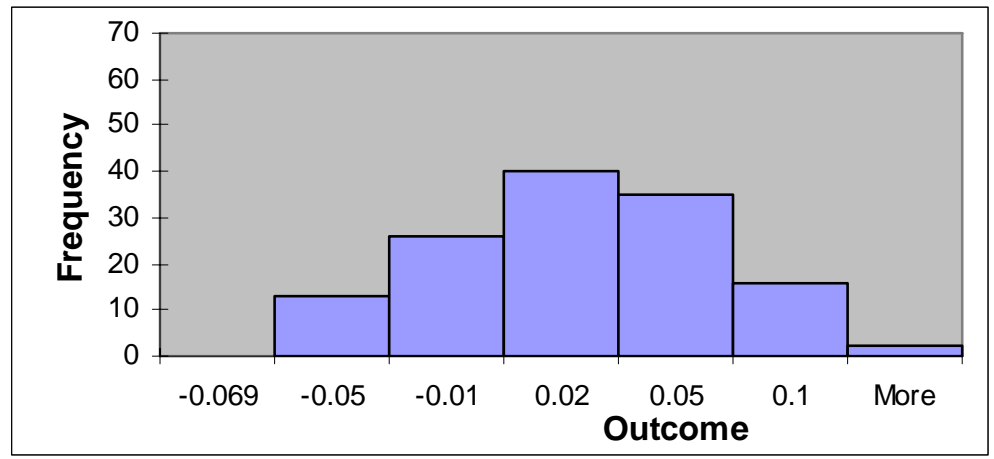

Fig. 5.2: The histogram of the return distribution of $P_{C V a R}$ for expected return $d_{1}=0.00927$.

In Fig. 5.3 below the histogram of the return distribution of $P_{v a r}$ for the same expected return $\mathrm{d}_{1}=0.00927$ is presented. This distribution is negatively skewed, with a long left tail, a short right tail and also a large probability of outcomes above the expected value; thus, there is a large probability of small gains. This distribution is concentrated around the expected value.

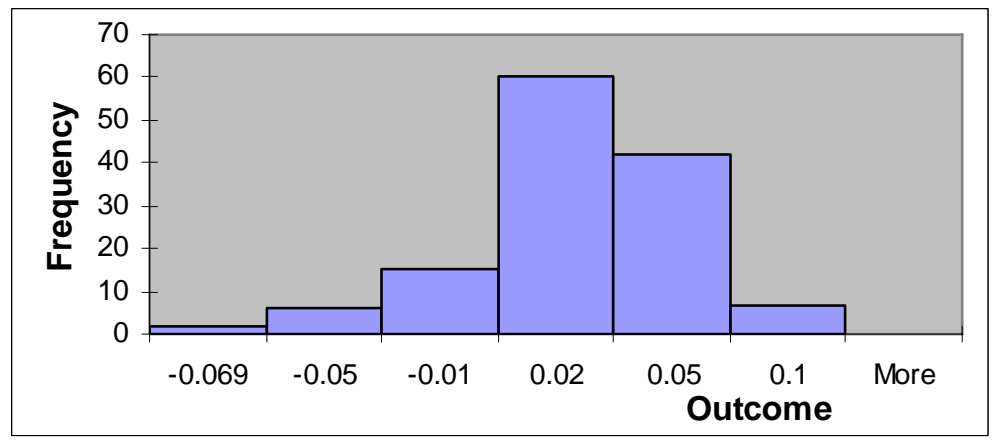

Fig. 5.3: The histogram of the return distribution of $P_{\text {var }}$ for expected return $d_{1}=0.00927$.

In Fig. 5.4 below the histogram of the return distribution of $\mathrm{P}_{1 / 4 \mathrm{CVaR}}$ for the same expected return $\mathrm{d}_{1}=0.00927$ is presented. This distribution has approximately the same shape as the return distribution of $\mathrm{P}_{\mathrm{var}}$ : concentrated around the expected value and with a large probability of outcomes just above the expected value. However, its left tail is shorter, due to the constraint imposed on the CVaR level, and thus the probability of large losses is reduced.

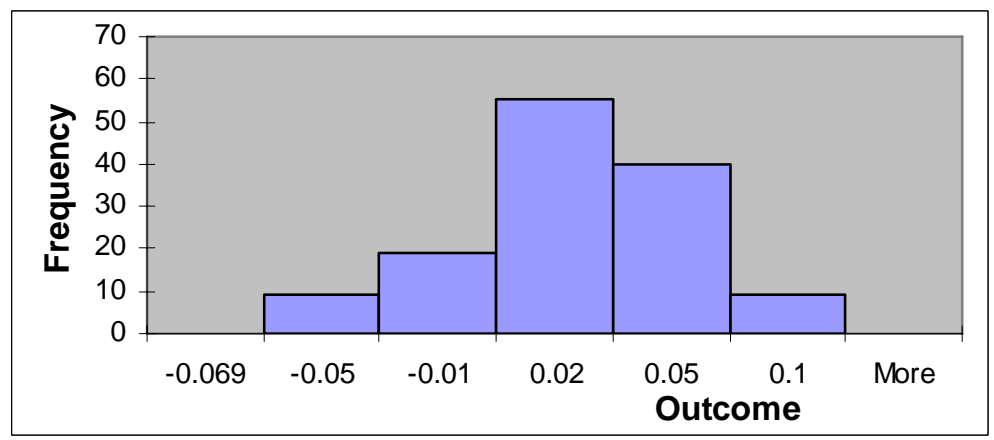

Fig. 5.4: The histogram of the return distribution of $P_{1 / 4 \mathrm{CVaR}}$ for expected return $d_{1}=0.00927$.

\subsection{Out-of-sample analysis}

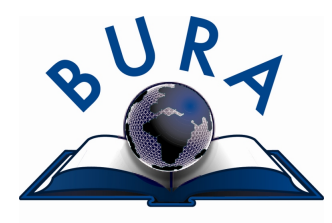


We analyse the performance of the portfolios described in the previous section over the next 18 time periods following the date of selection (January 2004-June 2005).

The portfolios that are non-efficient in either the mean-variance or the meanCVaR model, denoted by $\mathrm{P}_{1 / 4 \mathrm{CVaR}}, \mathrm{P}_{1 / 2 \mathrm{CVaR}}$ and $\mathrm{P}_{3 / 4 \mathrm{CVaR}}$, have an out-of-sample performance comparable to that of the mean-variance and the mean-CVaR efficient portfolios. It may be noted the generally good out-of-sample performance of the mean-CVaR portfolios and the somewhat poorer performance of the mean-variance portfolios, although the differences were not significant.

In general, the best out-of-sample parameters correspond to mean-CVaR portfolios, but for some levels of expected return, $\mathrm{P}_{1 / 4 \mathrm{CVaR}}$ had equally good or even better out-of-sample parameters (see Tables 5.2 and 5.3 below, with the best values in italic bold and the worse values enclosed by rectangles).

\begin{tabular}{|c|c|c|c|c|c|}
\hline & $\mathbf{P}_{\mathrm{CVaR}}$ & $\mathbf{P}_{1 / 4 \mathrm{CVaR}}$ & $\mathbf{P}_{1 / 2 \mathrm{CVaR}}$ & $P_{3 / 4 C V a R}$ & $\mathbf{P}_{\mathrm{var}}$ \\
\hline Mean & 0.016294 & 0.01472 & 0.013835 & 0.013556 & 0.01345 \\
\hline Median & 0.013106 & 0.015918 & 0.01456 & 0.012515[ & 0.011549 \\
\hline Standard Deviation & 0.029173 & 0.026514 & 0.025082 & 0.023893 & 0.022882 \\
\hline Minimum & -0.03494 & -0.03156 & -0.03316 & -0.02945 & -0.02491 \\
\hline Maximum & 0.052624 & 0.07282 & 0.071134 & 0.068515 & 0.066001 \\
\hline
\end{tabular}

Table 5.2: Ex-post parameters of the mean-variance-CVaR efficient portfolios with in-sample mean return $d_{1}=0.009268$.

\begin{tabular}{|c|c|c|c|c|c|}
\hline \multirow{3}{*}{$\begin{array}{l}\text { Mean } \\
\text { Median }\end{array}$} & $\mathbf{P}_{\mathrm{CVaR}}$ & $P_{1 / 4 C V a R}$ & $P_{1 / 2 \mathrm{CVaR}}$ & $P_{3 / 4 C V a R}$ & $\mathbf{P}_{\mathrm{var}}$ \\
\hline & 0.01133 & 0.012532 & 0.012342 & 0.012352 & 0.012342 \\
\hline & 0.010171 & 0.013783 & 0.013118 & 0.012365 & 0.01231 \\
\hline Standard Deviation & 0.028682 & 0.031943 & 0.03221 & 0.032159 & 0.032581 \\
\hline Minimum & -0.04247 & -0.03263 & -0.03614 & -0.03817 & -0.04004 \\
\hline Maximum & 0.081765 & 0.08752 & 0.082737 & 0.078024 & 0.07290 \\
\hline
\end{tabular}

Table 5.3: Ex-post parameters of the mean-variance-CVaR efficient portfolios with in-sample mean return $d_{3}=0.01880$.

Figure 5.5 presents the compound out-of-sample returns of the mean-variance-CVaR efficient portfolios with in-sample mean return $\mathrm{d}_{1}=0.009268$. $\mathrm{P}_{1 / 4 \mathrm{CVaR}}$ had a better out-of-sample performance than $\mathrm{P}_{\mathrm{CVaR}}$ in the first eight out-of-sample periods (January - August 2004) (moreover, $\mathrm{P}_{\mathrm{CVaR}}$ had a compound return less than one in February 2004, which means that its value fell below the amount invested). At the same time, $\mathrm{P}_{1 / 4 \mathrm{CV} a \mathrm{R}}$ had a better out-of-sample performance than $\mathrm{P}_{\mathrm{var}}$ in the last ten out-of-sample periods (September 2004 - June 2005). 


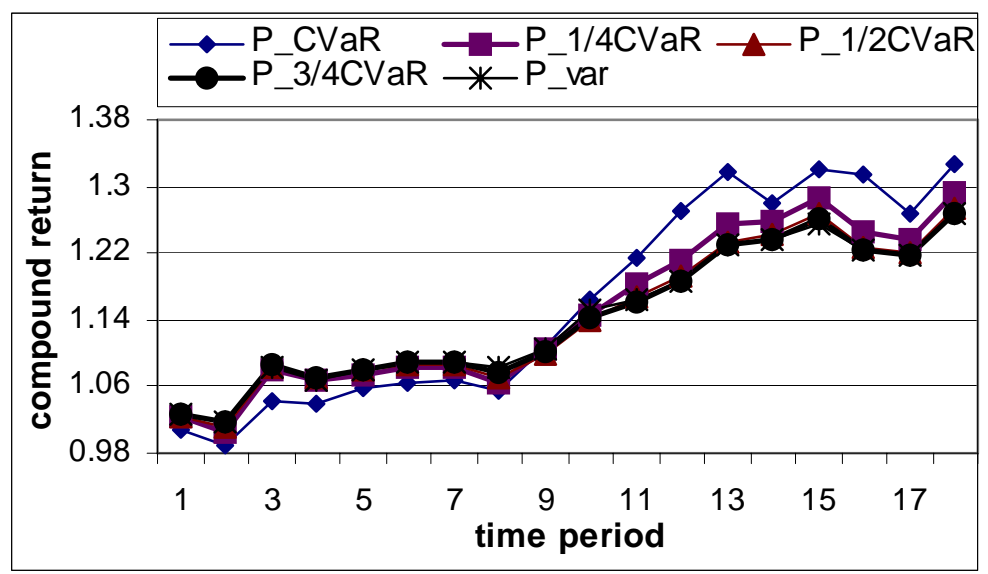

Fig. 5.5: Ex-post compounded returns of the mean-variance-CVaR efficient portfolios with insample mean return $d_{1}=0.009268$.

Figure 5.6 presents the compounded out-of-sample returns of the mean-varianceCVaR efficient portfolios with in-sample mean return $\mathrm{d}_{3}=0.01880 . \mathrm{P}_{1 / 4 \mathrm{CVaR}}$ had a better out-of-sample performance than both $\mathrm{P}_{\mathrm{CVaR}}$ and $\mathrm{P}_{\mathrm{var}}$, although the differences are small.

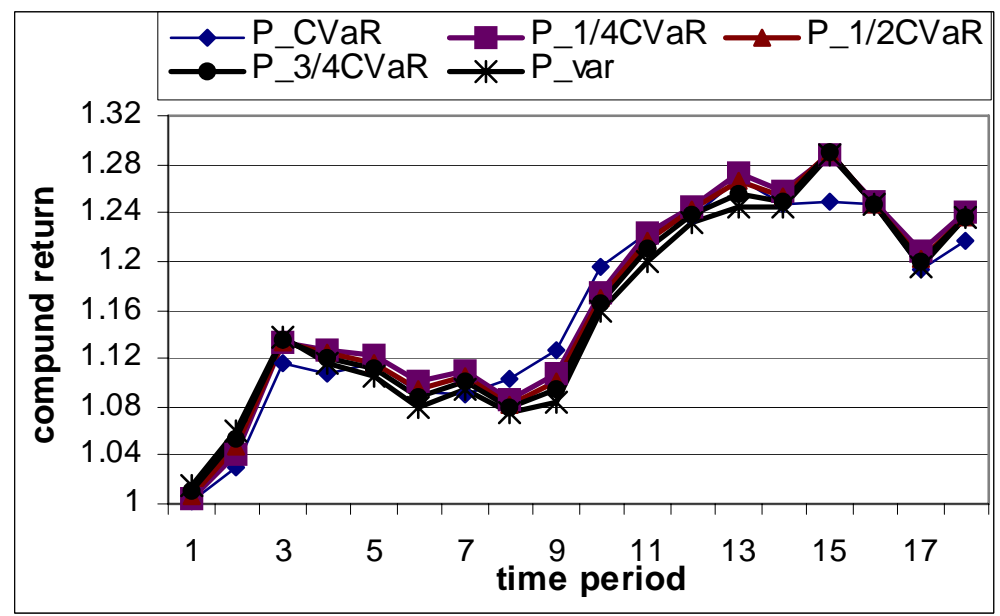

Fig. 5.6: Ex-post compounded returns of the mean-variance-CVaR efficient portfolios with insample mean return $d_{3}=\mathbf{0 . 0 1 8 8 0}$.

\section{Summary and Conclusions}

In this paper, we presented a model for portfolio selection, which selects a solution (distribution) on the basis of three parameters: the expected value, the variance and the CVaR at a specified confidence level. We called this model the mean-variance-CVaR model. The problem of selecting an efficient solution of this model is multi-objective: the expected value is maximised, while the variance and $\mathrm{CVaR}$ are minimised. We chose variance and CVaR mainly because they are well established risk measures that quantify risk from different perspectives: variance measures the deviation around the expected value while CVaR measures the average loss over a specified number of worst cases.

Computationally, the problem reduces to solving a single objective problem in which variance is minimised, while constraints are imposed on the expected value and

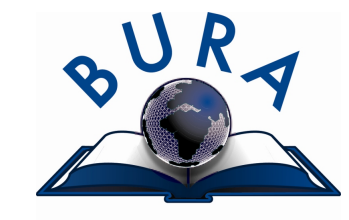


$\mathrm{CVaR}$. In the practice of portfolio selection, the random variables under consideration are usually represented as discrete and described by realisations under various scenarios. In this case, the problem is one of quadratic programming, thus routinely solved by standard available software. Having a constraint on CVaR rather than on the variance has advantages not only from a computational point of view. It is more natural to impose a maximum CVaR level than a maximum variance level, since $\mathrm{CVaR}$ represents the mean of the worst outcomes of a distribution.

Varying the right hand side of the constraints on the expected value and on $\mathrm{CVaR}$ such that these constraints are active produces all the efficient solutions of the mean-variance-CVaR model.

When solving the model for a fixed level of expected return, there is a range of efficient solutions. Plotted in a variance-CVaR space, they form a curve, with one end represented by the minimum variance portfolio (with the lowest CVaR), the other represented by the minimum $\mathrm{CVaR}$ portfolio (with the lowest variance).

The model was tested on a dataset drawn from the FTSE 100 index. Several levels of expected return were considered, and, for each level of expected return, five portfolios that were efficient in the mean-variance-CVaR model, were analysed: the minimum variance portfolio, the minimum CVaR portfolio and other three portfolios that were dominated in both mean-variance and mean-CVaR models. As expected, the best in-sample parameters concerning the left tail of distributions corresponded to mean-CVaR efficient portfolios: highest skewness, lowest kurtosis and highest maximum. However, the return distributions of mean-CVaR efficient portfolios have also the highest variances. In contrast, the mean-variance efficient portfolios have the return distributions with the lowest variance, but also with the "worst" left tail (as described by skewness, kurtosis, minimum and CVaR). The other portfolios, efficient only in the mean-variance-CVaR model, improve on the left tail of the mean-variance efficient distributions: they have higher skewness, lower kurtosis higher maximum and higher $\mathrm{CVaR}$. In some cases, this improvement comes at the expense of only a marginal increase in variance. The out-of-sample performances of these portfolios are comparable to those of the mean-variance and mean-CVaR efficient portfolios. In two out of five cases, such a portfolio achieved the highest mean of out-of-sample returns and in almost all cases led to the highest maximum of out-of-sample returns.

As a final remark, it may be noted that the proposed model does not dismiss mean-variance or mean-CVaR models, but on the contrary, it "embeds" them. Most of the mean-variance and the mean-CVaR efficient solutions are particular solutions of the proposed model. For example, a mean-variance efficient solution is not a solution of the proposed model only if there is another mean-variance efficient solution with the same mean and variance but with lower CVaR. Likewise, from the set of mean$\mathrm{CVaR}$ efficient solutions with a specified mean return, only the one(s) with the lowest variance is solution of the proposed model. Thus, the proposed model makes a "positive" discrimination between mean-variance and mean-CVaR efficient solutions. In addition, the mean-variance-CVaR model has a range of solutions that are normally discarded by both mean-variance and mean-CVaR model. These solutions may bring an improvement in the distribution, in the case when the CVaR of a mean-variance efficient portfolio is considered to be unacceptably large. They represent a compromise between regulators' requirements for short tails and classical fund managers' requirements for small variance. In making the final choice, the personal preference of the decision-maker plays a key role. 


\section{REFERENCES}

ACERBI, C., D. TASCHE (2002): On the Coherence of Expected Shortfall, Journal of Banking and Finance 26, 1487-1503.

ARTZNER, P., F. DELBAEN, J.M. EBER, D. HEATH (1999): Coherent Measures of Risk, Mathematical Finance, 9 (3), pp 203-228.

DAS, I. And J. DENNIS (1997): A Closer Look at the Drawbacks of Minimising Weighted Sums of Objectives for Pareto Set Generation in Multicriteria Optimisation problems, Structural Optimisation, 14(1), 63-69.

FISHBURN, P.C. (1977). Mean- Risk Analysis with Risk Associated with Below Target Returns, The American Economic Review 67, 116-126.

HAIMES, Y. Y., LASDON, L. S. and WISMER, D. A. (1971): On a bicriterion formulation of the problems of integrated system identification and system optimization, IEEE Trans. on Systems, Man and Cybernetics 1, 296-297.

HARVEY, C., LIECHTY, R., John, LIECHTY, M. W. and MUELLER, P. (2003) Portfolio Selection With Higher Moments. Working Paper, Duke University. http://ssrn.com/abstract=634141

ILOG (2003): CPLEX 9.0, User’s manual, ILOG SA, Gentilly, France.

JAHN, J. (1985): Some characterizations of the optimal solutions of a vector optimization problem, OR Spektrum, 7, 7-17.

JORION, P. (2003): Portfolio Optimisation with Tracking-Error Constraints, Financial Analysts Journal, 59(5), 70-82.

KONNO, H and H. YAMAZAKI. (1991) Mean absolute deviation portfolio optimization model and its applications to Tokyo stock market, Management Science, 37, 519-531.

KONNO, H., SHIRAKAWA, H. and H. YAMAZAKI. (1993): A mean-absolute deviation-skewness portfolio optimisation model, Annals of Operations Research 45, 205-220.

KONNO, H and SUZUKI, K. (1995): A mean-variance-skewness portfolio optimisation model, Journal of Operations Research Society of Japan, 38(2), 173-187.

KROKHMAL, P., PALMQUIST, J. and URYASEV, S. (2002): Portfolio Optimisation with Conditional Value-at-Risk Objective and Constraints, Journal of Risk, 4(2), 43-68.

LARSEN, N., MAUSSER, H. and URYASEV, S. (2002): Algorithms for Optimisation of Value-at-Risk, in P. Pardalos and V.K. Tsitsiringos (editors), 
Financial Engineering, E-Commerce and Supply Chain, Kluwer Academic Publishers (Norwell) pp. 129-157.

LAURENT, J. P. (2003): Sensitivity Analysis of Risk Measures for Discrete Distributions, available from:

http://laurent.jeanpaul.free.fr/var_risk_measure_sensitivity.pdf

LEYFFER, S. and PANG, J.S. (2005): On the Global Minimization of the Value-atRisk, available from: http://www.citebase.org/cgi-

bin/citations?id=oai:arXiv.org:math/0401063

LUENBERGER, D. G. (1998): Investment Science, Oxford University Press, New York.

MAKOWSKI, M. and A.P. WIERZBICKI (2003): Modelling Knowledge. Model Based Decision Support and Soft Computations, Applied Decision Support with Soft Computing, 124, 3-60, Springer-Verlag, Berlin, NY; also available at:

www.iiasa.ac.at/ marek/pubs.

MARKOWITZ, H. M. (1952): Portfolio Selection, Journal of Finance, 7(1), 77-91.

MAXIMAL SOFTWARE INCORPORATION (2000): MPL Modelling System, Release 4.11, Arlington, Virginia, USA.

OGRYCZAK, W. (2000): Multiple Criteria Linear Programming Model for Portfolio Selection, Annals of Operations Research, 97, 143-162.

OGRYCZAK, W. (2002): Multiple Criteria Optimization and Decisions under Risk, Control and Cybernetics, 31, 975-1003.

OGRYCZAK, W., A. RUSZCZYNSKI (1999): From Stochastic Dominance to Mean- Risk Models: Semideviations as Risk Measures, European Journal of Operational Research, 116, 33-50.

OGRYCZAK, W., A. RUSZCZYNSKI (2001): On Consistency of Stochastic Dominance and Mean- Semideviations Models, Mathematical Programming, 89, 217 232.

OGRYCZAK, W., A. RUSZCZYNSKI (2002): Dual Stochastic Dominance and Related Mean- Risk Models, SIAM Journal on Optimization, 13, 60-78.

PFLUG, G (2000): Some remarks on the Value-at-Risk and the Conditional Value-atRisk, In: Probabilistic Constrained Optimisation: Methodology and Applications (ed. S Uryasev), available from www.gloriamundi.org.

ROCKAFELLER, R.T., S. URYASEV (2000): Optimization of Conditional Value-atRisk, Journal of Risk 2 (2000), 21-42. 
ROCKAFELLER, R.T., S. URYASEV (2002): Conditional Value-at-Risk for general loss distributions, Journal of Banking and Finance, 26/7, 2002, 1443-1471.

STEUER, R. (1986): Multiple Criteria Optimization - Theory, Computation and Application, John Wiley \& Sons, New York.

STEUER, R. and NA, P. (2003): Multiple criteria decision making combined with finance: A categorized bibliographic study, European Journal of Operational Research, 150, 496-515.

TASCHE, D. (2002): Expected Shortfall and Beyond, Journal of Banking and Finance 26(7), 1519-1533.

WANG, J. (2000): Mean-Variance-VaR Based Portfolio Optimisation, available from www.gloriamundi.org.

WIERZBICKI, A. (1998): Reference Point Methods in Vector Optimization and Decision Support, IIASA Interim Report IR-98-017.

YITZHAKI, S. (1982): Stochastic Dominance, Mean Variance and Gini’s Mean Difference, American Economic Review 72 178-185.

\section{Appendix A. The general case of a positive semi-definite covariance matrix}

Here we describe a method of producing the entire efficient frontier of the meanvariance-CVaR model for the general case when the covariance matrix of returns is positive semi-definite. In this case, the minimisation of variance over a convex set may not have a unique optimal solution. Thus, when using the optimisation problem (P2) as described in Section 4.4, we may obtain solutions that are Pareto dominated in

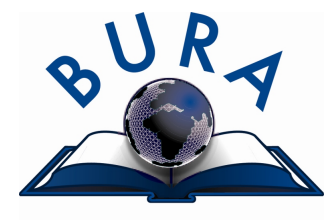


$(\mathrm{MVC})^{8}$. However, we can still use (P2) to produce the entire set of Pareto nondominated solutions of (MVC), provided the right hand sides $\mathrm{d}$ and $\mathrm{z}$ for the mean and CVaR constraints are chosen as described below.

The level $d$ for the expected value must lie in the interval [ $d^{\prime}{ }_{\min }, d_{\max }$ ] where $d_{\max }$ is the maximum possible expected return (as presented in Section 4.4). We define $d^{\prime}{ }_{\text {min }}=\max \left\{d^{\prime}{ }_{\text {minvar }}, d_{\text {minCVaR }}\right\}$, where $d_{\text {minvar }}$ and $d_{\text {minCVaR }}$ are the expected returns of the minimum variance portfolio (mean-variance efficient) and minimum CVaR portfolio (mean-CVaR efficient) respectively. $d_{\text {minCVaR }}$ may be found as described in Section 4.4. The expected return of the minimum variance portfolio $\mathrm{d}^{\text {' }}$ minvar cannot be determined so straightforward as for the case of a positive definite covariance matrix. We cannot just minimise variance over the whole feasible set $\mathcal{A}$ (with no constraints on the mean) since there may be different optimal solutions to this problem, with the same (optimal) variance but with different expected returns. Among these solutions that globally minimise variance, we consider only the one with the maximum expected return. To obtain this solution, we first solve the problem:

$\min \sigma^{2}(x)$

Subject to: $\mathrm{x} \in \mathcal{A}$.

Denote the optimum value of this problem by $\sigma_{\min }$.

In order to find the specific optimal solution of this problem with the maximum possible expected return, we propose a convex program with quadratic constraint:

$\max E(x)$

Subject to: $\sigma^{2}(x) \leq \sigma_{\min }$ $\mathrm{x} \in \mathcal{A}$.

The optimal value of the above optimisation problem is $\mathrm{d}_{\text {'minvar. }}$.

Furthermore, for a specific $d \in\left[\mathrm{d}^{\prime}{ }_{\text {min }}, \mathrm{d}_{\text {max }}\right]$, the right hand side for the CVaR constraint $\mathrm{z}$ must lie in the interval $\left[\mathrm{z}_{\mathrm{d}, \min }, \mathrm{z}_{\mathrm{d} \text {,max }}{ }\right.$; $\mathrm{z}_{\mathrm{d} \text {,min }}$ is the best (minimum) $\mathrm{CVaR}_{\alpha}$ level for the expected return $d$ and may be found as described in Section 4.4. $\mathrm{z}_{\mathrm{d} \text {,max }}$ is the minimum $\mathrm{CVaR}_{\alpha}$ level of the mean-variance efficient portfolios with expected return $\mathrm{d}^{9}$.

In order to determine $\mathrm{z}_{\mathrm{d} \text {,max }}$, one may solve two optimisation problems. Firstly, the optimal variance for the expected return d (denoted by $\sigma_{d}^{2}$ ) may be found as the optimal value of the objective function in the problem:

$\min \sigma^{2}(x)$

Subject to: $\mathrm{E}(\mathrm{x}) \geq \mathrm{d}$

$\mathrm{x} \in \mathcal{A}$.

Secondly, $z_{d, \max }$ may be found as the optimal value of the objective function in the problem:

\footnotetext{
${ }^{8}$ For example, multiple optimal solutions of (P2) may have the same variance, the same expected return but different CVaRs; only the one with the lowest CVaR is Pareto efficient in (MVC).

${ }^{9}$ In case there are several mean-variance efficient portfolios with expected return d, with different CVaR levels, only the portfolio with the lowest CVaR is efficient in the (MVC) model; its CVaR level is denoted by $\mathrm{z}_{\mathrm{d}, \mathrm{max}}$.
} 
$\operatorname{Min} \mathrm{F}_{\alpha}(\mathrm{x}, \mathrm{v})$

Subject to: $\mathrm{E}(\mathrm{x}) \geq \mathrm{d}$

$$
\begin{aligned}
& \sigma^{2}(x) \leq \sigma^{2}{ }_{d} \\
& x \in \mathcal{A}, v \in R .
\end{aligned}
$$

Proposition A.1: Consider the optimisation problem

(P1): $\min \sigma^{2}(x)$

Subject to: $C \operatorname{VaR}(x) \leq \mathrm{z}$

$$
\begin{aligned}
& \mathrm{E}(\mathrm{x}) \geq \mathrm{d} \\
& \mathrm{x} \in \mathcal{A} .
\end{aligned}
$$

If $\mathrm{x}^{*}$ is an optimal solution of (P1) for $\mathrm{d} \in\left[\mathrm{d}_{\text {min }}, \mathrm{d}_{\max }\right]$ and $\mathrm{z} \in\left[\mathrm{z}_{\mathrm{d}, \min }, \mathrm{z}_{\mathrm{d}, \max }\right]$ (as described above), then $\mathrm{X}^{*}$ is Pareto efficient in (MVC).

Proof: Assume that $\mathrm{x}^{*}$ is not Pareto efficient in (MVC). Denote by $\mathrm{x}^{\prime}$ a feasible solution of (MVC) that Pareto dominates $x^{*}$. This means that $\sigma^{2}\left(x^{\prime}\right) \leq \sigma^{2}\left(x^{*}\right)$, $\mathrm{CVaR}_{\alpha}\left(\mathrm{x}^{\prime}\right) \leq \mathrm{CVaR} \alpha\left(\mathrm{x}^{*}\right) \leq \mathrm{z}$ and $\mathrm{E}\left(\mathrm{x}^{\prime}\right) \geq \mathrm{E}\left(\mathrm{x}^{*}\right) \geq \mathrm{d}$ with at least one strict inequality. Thus, $x^{\prime}$ is a feasible solution of (P1). The case $\sigma^{2}\left(x^{\prime}\right)<\sigma^{2}\left(x^{*}\right)$ is excluded since this contradicts the fact that $x^{*}$ is an optimal solution of (P1). It only remains the possibility that $\mathrm{x}^{\prime}$ and $\mathrm{x}^{*}$ are both optimal solutions of (P1) and

$\mathrm{CVaR}_{\alpha}\left(\mathrm{x}^{\prime}\right)<\mathrm{CVaR}_{\alpha}\left(\mathrm{x}^{*}\right) \leq \mathrm{z}$ or $\mathrm{E}\left(\mathrm{x}^{\prime}\right)>\mathrm{E}\left(\mathrm{x}^{*}\right) \geq \mathrm{d}$.

Consider first the case $\mathrm{CVaR}_{\alpha}\left(\mathrm{x}^{\prime}\right)<\mathrm{CVaR}_{\alpha}\left(\mathrm{x}^{*}\right) \leq \mathrm{z}$; thus, $\mathrm{x}^{\prime}$ is an optimal solution of (P1) and the constraint $C \operatorname{VaR}_{\alpha}(x) \leq \mathrm{z}$ is not binding. Since (P1) is a convex optimisation problem, it follows that $\mathrm{x}$ ' is an optimal solution of the "reduced" problem, obtained from (P1) by removing the constraint on CVaR:

$\left(\mathrm{P} 1_{\text {red }}\right)$ : $\min \sigma^{2}(x)$

$$
\begin{array}{r}
\text { Subject to: } \mathrm{E}(\mathrm{x}) \geq \mathrm{d} \\
\mathrm{x} \in \mathcal{A} .
\end{array}
$$

This means that both $x^{\prime}$ and $x^{*}$ are mean-variance efficient portfolios with expected return $\mathrm{d} \in\left[\mathrm{d}{ }_{\text {min }}, \mathrm{d}_{\max }\right]$. Thus, we have two mean-variance efficient solutions with the same variance, the same expected return $\mathrm{d}$ but different $\mathrm{CV}$ aRs.

$\mathrm{CVaR}_{\alpha}\left(\mathrm{x}^{\prime}\right)<\mathrm{CVaR}_{\alpha}\left(\mathrm{x}^{*}\right) \leq \mathrm{z} \leq \mathrm{z}_{\mathrm{d} \text {,max }}$. However, $\mathrm{z}_{\mathrm{d} \text {,max }}$ is, by construction, the lowest possible $\mathrm{CVaR}$ of a mean-variance efficient portfolio with mean return $\mathrm{d}$ and we have a contradiction.

Obviously the constraint $\mathrm{E}(\mathrm{x}) \geq \mathrm{d}$ in (P1) is binding for $\mathrm{d} \in\left[\mathrm{d}{ }^{\prime}{ }_{\text {min }}, \mathrm{d}_{\text {max }}\right]$; thus, the case $\mathrm{E}\left(\mathrm{x}^{\prime}\right)>\mathrm{E}\left(\mathrm{x}^{*}\right) \geq \mathrm{d}$ is also impossible and this ends the proof.

Thus, when the right hand sides $\mathrm{d}$ and $\mathrm{v}$ are chosen as above, the constraints on CVaR and on mean are active.

It was shown in Section 4.2 that the constraint $C V a R_{\alpha}(x) \leq \mathrm{z}$ can be replaced with the constraint $F_{\alpha}(x, v) \leq z, v \in R$ and thus the problem (P2), equivalent to (P1), is obtained:

(P2): $\min \sigma^{2}(x)$

$$
\begin{array}{cl}
\text { Subject to: } & F_{\alpha}(x, v) \leq \mathrm{z} \\
& \mathrm{E}(\mathrm{x}) \geq \mathrm{d}
\end{array}
$$




$$
\mathrm{x} \in \mathcal{A}, \mathrm{v} \in \mathrm{R}
$$

Solving problem (P2) with d varying between $\mathrm{d}_{\text {min }}$ and $\mathrm{d}_{\max }$ and $\mathrm{z}$ varying between $z_{d, \min }$ and $z_{d, \text { max }}$ as described above produces all the efficient solutions of the mean-variance-CVaR model.

\section{Appendix B. The composition of efficient portfolios}

\begin{tabular}{|c|c|c|c|c|c|}
\hline asset index & $\mathbf{P}_{\text {CVaR }}$ & $\mathbf{P}_{1 / 4 \text { CVaR }}$ & $\mathbf{P}_{1 / 2 \text { CVaR }}$ & $\mathbf{P}_{3 / 4 C V a R}$ & $\mathbf{P}_{\text {var }}$ \\
\hline 4 & 0.028 & 0.074 & 0.059 & 0.049 & 0.047 \\
\hline 5 & 0.194 & 0.050 & 0.034 & 0.027 & 0.024 \\
\hline 11 & 0 & 0.055 & 0.068 & 0.065 & 0.052 \\
\hline 13 & 0 & 0.059 & 0.070 & 0.075 & 0.072 \\
\hline 16 & 0 & 0.013 & 0.029 & 0.025 & 0.018 \\
\hline 17 & 0 & 0.005 & 0.026 & 0.046 & 0.048 \\
\hline 21 & 0 & 0 & 0.008 & 0.009 & 0.007 \\
\hline 24 & 0 & 0 & 0 & 0.008 & 0.023 \\
\hline 25 & 0 & 0.018 & 0.026 & 0.037 & 0.045 \\
\hline 27 & 0.004 & 0.049 & 0.076 & 0.076 & 0.071 \\
\hline 40 & 0.208 & 0.093 & 0.081 & 0.064 & 0.067 \\
\hline 42 & 0 & 0.017 & 0.044 & 0.056 & 0.051 \\
\hline 43 & 0.061 & 0.086 & 0.067 & 0.061 & 0.056 \\
\hline 44 & 0.026 & 0.075 & 0.059 & 0.046 & 0.052 \\
\hline 45 & 0.073 & 0.078 & 0.066 & 0.052 & 0.042 \\
\hline 48 & 0 & 0 & 0 & 0 & 0.007 \\
\hline 63 & 0 & 0 & 0 & 0 & 0.015 \\
\hline 64 & 0 & 0 & 0 & 0.002 & 0.005 \\
\hline 65 & 0.025 & 0.100 & 0.066 & 0.057 & 0.039 \\
\hline 66 & 0 & 0 & 0.006 & 0.045 & 0.064 \\
\hline 69 & 0.171 & 0.073 & 0.035 & 0.010 & 0 \\
\hline 70 & 0 & 0 & 0.033 & 0.056 & 0.064 \\
\hline 72 & 0 & 0.026 & 0.051 & 0.059 & 0.069 \\
\hline 73 & 0.211 & 0.129 & 0.094 & 0.077 & 0.063 \\
\hline
\end{tabular}

Table 1: The portfolio weights of the efficient portfolios for $d_{1}=0.009268$.

\begin{tabular}{|c|c|c|c|c|c|}
\hline asset index & $\mathbf{P}_{\text {CVaR }}$ & $\mathbf{P}_{1 / 4 \text { CVaR }}$ & $\mathbf{P}_{1 / 2 \text { CVaR }}$ & $\mathbf{P}_{3 / 4 C V a R}$ & $\mathbf{P}_{\text {var }}$ \\
\hline 4 & 0.095 & 0.106 & 0.099 & 0.087 & 0.082 \\
\hline 5 & 0.140 & 0.075 & 0.059 & 0.058 & 0.059 \\
\hline 10 & 0.044 & 0 & 0 & 0 & 0 \\
\hline 13 & 0 & 0.067 & 0.071 & 0.080 & 0.084 \\
\hline 16 & 0.032 & 0.036 & 0.031 & 0.015 & 0.011 \\
\hline 17 & 0 & 0.043 & 0.064 & 0.073 & 0.075 \\
\hline 21 & 0.011 & 0.042 & 0.043 & 0.046 & 0.050 \\
\hline 24 & 0 & 0 & 0 & 0.001 & 0.014 \\
\hline
\end{tabular}

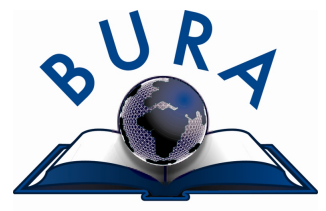




\begin{tabular}{|c|c|c|c|c|c|}
25 & 0.019 & 0.007 & 0.021 & 0.025 & 0.020 \\
\hline 27 & 0 & 0.045 & 0.039 & 0.028 & 0.020 \\
\hline 28 & 0.074 & 0.031 & 0 & 0 & 0 \\
\hline 40 & 0 & 0.060 & 0.064 & 0.047 & 0.033 \\
\hline 42 & 0 & 0 & 0.000 & 0.005 & 0.006 \\
\hline 43 & 0 & 0.031 & 0.006 & 0 & 0 \\
\hline 44 & 0.056 & 0.111 & 0.100 & 0.080 & 0.082 \\
\hline 45 & 0.179 & 0.165 & 0.133 & 0.116 & 0.103 \\
\hline 48 & 0 & 0 & 0 & 0.015 & 0.020 \\
\hline 56 & 0 & 0 & 0.013 & 0.029 & 0.039 \\
\hline 58 & 0.024 & 0.009 & 0.017 & 0.027 & 0.032 \\
\hline 63 & 0 & 0.005 & 0.034 & 0.043 & 0.048 \\
\hline 65 & 0 & 0 & 0.003 & 0.019 & 0.025 \\
\hline 66 & 0 & 0 & 0.028 & 0.042 & 0.050 \\
\hline 69 & 0.079 & 0 & 0 & 0 & 0 \\
\hline 70 & 0 & 0 & 0.036 & 0.054 & 0.061 \\
\hline 73 & 0.246 & 0.164 & 0.140 & 0.110 & 0.085 \\
\hline
\end{tabular}

Table 2: The portfolio weights of the efficient portfolios for $d_{2}=\mathbf{0 . 0 1 4 0 3}$.

\begin{tabular}{|c|c|c|c|c|c|}
\hline asset index & $\mathbf{P}_{\text {CVaR }}$ & $\mathbf{P}_{1 / 4 \text { CVaR }}$ & $\mathbf{P}_{1 / 2 \text { CVaR }}$ & $\mathbf{P}_{3 / 4 \mathrm{CVaR}}$ & $\mathbf{P}_{\text {var }}$ \\
\hline 4 & 0.143 & 0.128 & 0.124 & 0.123 & 0.119 \\
\hline 5 & 0.091 & 0.024 & 0.043 & 0.056 & 0.064 \\
\hline 13 & 0 & 0 & 0 & 0 & 0.024 \\
\hline 16 & 0 & 0 & 0.002 & 0.022 & 0.036 \\
\hline 17 & 0 & 0.028 & 0.056 & 0.062 & 0.061 \\
\hline 21 & 0.038 & 0.094 & 0.107 & 0.119 & 0.130 \\
\hline 44 & 0.062 & 0.161 & 0.135 & 0.130 & 0.124 \\
\hline 45 & 0.298 & 0.228 & 0.215 & 0.187 & 0.165 \\
\hline 48 & 0 & 0 & 0.000 & 0 & 0 \\
\hline 56 & 0.045 & 0.066 & 0.085 & 0.103 & 0.118 \\
\hline 58 & 0.084 & 0.080 & 0.075 & 0.073 & 0.070 \\
\hline 63 & 0 & 0.023 & 0.024 & 0.016 & 0.004 \\
\hline 73 & 0.239 & 0.164 & 0.125 & 0.093 & 0.065 \\
\hline 76 & 0 & 0.004 & 0.010 & 0.016 & 0.020 \\
\hline
\end{tabular}

Table 3: The portfolio weights of the efficient portfolios for $d_{3}=0.0188$.

\begin{tabular}{|c|c|c|c|c|c|}
\hline asset index & $\mathbf{P}_{\text {CVaR }}$ & $\mathbf{P}_{1 / 4 \text { CVaR }}$ & $\mathbf{P}_{1 / 2 \text { CVaR }}$ & $\mathbf{P}_{\text {3/4CVaR }}$ & $\mathbf{P}_{\text {var }}$ \\
\hline 4 & 0.044 & 0.154 & 0.147 & 0.135 & 0.134 \\
\hline 21 & 0.262 & 0.175 & 0.190 & 0.203 & 0.218 \\
\hline 44 & 0 & 0 & 0.017 & 0.032 & 0.045 \\
\hline 45 & 0.381 & 0.276 & 0.256 & 0.246 & 0.218 \\
\hline 56 & 0 & 0.103 & 0.138 & 0.174 & 0.204 \\
\hline 58 & 0.171 & 0.181 & 0.168 & 0.154 & 0.145 \\
\hline 73 & 0.141 & 0.090 & 0.056 & 0.021 & 0 \\
\hline 76 & 0 & 0.021 & 0.027 & 0.035 & 0.036 \\
\hline
\end{tabular}

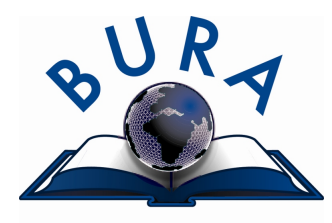


Table 4: The portfolio weights of the efficient portfolios for $\mathbf{d}_{\mathbf{4}}=\mathbf{0 . 0 2 3 5 7}$.

\begin{tabular}{|c|c|c|c|c|c|}
\hline asset index & $\mathbf{P}_{\text {CVaR }}$ & $\mathbf{P}_{\mathbf{1 / 4 C V a R}}$ & $\mathbf{P}_{\mathbf{1 / 2} \text { CVaR }}$ & $\mathbf{P}_{\mathbf{3} / \mathbf{4} \text { CVaR }}$ & $\mathbf{P}_{\text {var }}$ \\
\hline 4 & 0 & 0 & 0 & 0.013 & 0.015 \\
\hline 21 & 0.298 & 0.331 & 0.335 & 0.323 & 0.327 \\
\hline 45 & 0.234 & 0.158 & 0.097 & 0.056 & 0.016 \\
\hline 56 & 0 & 0.071 & 0.136 & 0.159 & 0.195 \\
\hline 58 & 0.469 & 0.441 & 0.429 & 0.418 & 0.405 \\
\hline 76 & 0 & 0 & 0.003 & 0.032 & 0.042 \\
\hline
\end{tabular}

Table 5: The portfolio weights of the efficient portfolios for $d_{5}=\mathbf{0 . 0 2 8 3 3}$.

For the highest level of expected return $\mathrm{d}_{6}=\mathrm{d}_{\max }=0.0331$, the efficient portfolio consists of the asset no 58 .

\section{Appendix C. The in-sample parameters for the return distributions of efficient portfolios}

\begin{tabular}{|c|c|c|c|c|c|}
\hline & $\mathbf{P}_{\mathrm{CVaR}}$ & $\mathbf{P}_{1 / 4 \mathrm{CVaR}}$ & $\mathbf{P}_{1 / 2 \mathrm{CVaR}}$ & $\mathbf{P}_{3 / 4 \mathrm{CVaR}}$ & $\mathbf{P}_{\mathrm{var}}$ \\
\hline Median & 0.010905 & 0.009989 & 0.010678 & 0.011774 & 0.011348 \\
\hline Standard Deviation & 0.039557 & 0.032288 & 0.030899 & 0.030186 & 0.030006 \\
\hline Skewness & 0.175763 & -0.43318 & -0.59261 & -0.75996 & -0.89894 \\
\hline Kurtosis & -0.16328 & 0.214433 & 0.763715 & 1.35481 & 1.964419 \\
\hline Minimum & -0.05813 & -0.06857 & -0.08198 & -0.09601 & -0.10946 \\
\hline Maximum & 0.128209 & 0.085995 & 0.084375 & 0.081927 & 0.077194 \\
\hline
\end{tabular}

Table 6: In-sample parameters for the return distributions of efficient portfolios in the meanvariance-0.01CVaR model with expected return $d_{1}=0.009268$.

\begin{tabular}{|l|crrrr|}
\hline \multirow{4}{*}{ Median } & $\mathbf{P}_{\text {CVaR }}$ & $\mathbf{P}_{1 / 4 \mathrm{CVaR}}$ & $\mathbf{P}_{1 / 2 \mathrm{CVaR}}$ & $\mathbf{P}_{\mathbf{3} 4 \mathrm{CVaR}}$ & \multicolumn{1}{c|}{$\mathbf{P}_{\text {var }}$} \\
\cline { 2 - 6 } Standard Deviation & 0.009982 & 0.016801 & 0.016398 & 0.017359 & $\mathbf{0 . 0 1 7 6}$ \\
\cline { 2 - 6 } Skewness & 0.043277 & 0.035516 & 0.034453 & 0.03398 & $\mathbf{0 . 0 3 3 8 5 2}$ \\
\cline { 2 - 6 } Kurtosis & $\mathbf{0 . 2 3 8 3 1 7}$ & -0.5367 & -0.64824 & -0.75897 & -0.87193 \\
Minimum & $\mathbf{0 . 1 0 0 6 8 9}$ & 0.329636 & 0.799505 & 1.213484 & 1.633637 \\
Maximum & $-\mathbf{0 . 0 7 0 5 6}$ & -0.07906 & -0.08756 & -0.09606 & -0.10498 \\
\hline
\end{tabular}

Table 7: In-sample parameters for the return distributions of efficient portfolios in the meanvariance-0.01CVaR model with expected return $d_{2}=0.014034$. 


\begin{tabular}{|l|ccccc|}
\hline \multirow{4}{*}{ Median } & $\mathbf{P}_{\text {CVaR }}$ & $\mathbf{P}_{\mathbf{1 / 4 C V R}}$ & $\mathbf{P}_{\mathbf{1 / 2 C V a R}}$ & $\mathbf{P}_{\mathbf{3} 4 \mathrm{CVaR}}$ & $\mathbf{P}_{\mathrm{var}}$ \\
\cline { 2 - 6 } Standard Deviation & 0.019982 & 0.021909 & 0.021945 & $\mathbf{0 . 0 2 2 4 5 3}$ & 0.02225 \\
\cline { 2 - 6 } Skewness & 0.051467 & 0.045116 & 0.043917 & 0.043138 & $\mathbf{0 . 0 4 2 8 6 9}$ \\
\cline { 2 - 6 } Kurtosis & $\mathbf{0 . 1 0 5 1 3 8}$ & -0.27928 & -0.35782 & -0.44374 & -0.50531 \\
Minimum & 0.816632 & $\mathbf{0 . 5 8 8 5 8 2}$ & 0.748811 & 1.016336 & 1.309189 \\
Maximum & $-\mathbf{0 . 0 9 1 8 6}$ & -0.10046 & -0.11094 & -0.12183 & -0.13216 \\
\hline
\end{tabular}

Table 8: In-sample parameters for the return distributions of efficient portfolios in the meanvariance-0.01CVaR model with expected return $d_{3}=0.018801$

\begin{tabular}{|c|c|c|c|c|c|}
\hline & $\mathbf{P}_{\mathrm{CVaR}}$ & $\mathbf{P}_{1 / 4 \mathrm{CV} V \mathrm{R}}$ & $\mathbf{P}_{1 / 2 \mathrm{CVaR}}$ & $\mathbf{P}_{3 / 4 \mathrm{CVaR}}$ & $\mathbf{P}_{\mathrm{var}}$ \\
\hline Median & 0.026665 & 0.02185 & 0.023582 & 0.022484 & 0.023786 \\
\hline Standard Deviation & 0.071333 & 0.061135 & 0.059382 & 0.058374 & 0.058031 \\
\hline Skewness & 0.595438 & -0.12047 & -0.23122 & -0.30692 & -0.36555 \\
\hline Kurtosis & 3.354617 & 0.816052 & 0.797705 & 0.808283 & 0.834841 \\
\hline Minimum & -0.12247 & -0.13142 & -0.14231 & -0.1528 & -0.16327 \\
\hline Maximum & 0.367729 & 0.204922 & 0.181086 & 0.162425 & 0.159635 \\
\hline
\end{tabular}

Table 9: In-sample parameters for the return distributions of efficient portfolios in the meanvariance-0.01CVaR model with the expected return $d_{4}=0.023567$.

\begin{tabular}{|l|ccccc|}
\hline & $\mathbf{P}_{\text {CVaR }}$ & $\mathbf{P}_{1 / 4 \text { CVaR }}$ & $\mathbf{P}_{1 / 2 \text { CVaR }}$ & $\mathbf{P}_{\text {3/4CVaR }}$ & $\mathbf{P}_{\text {var }}$ \\
\hline Median & $\mathbf{0 . 0 3 5 2 5 6}$ & 0.032523 & 0.027021 & 0.023606 & 0.022036 \\
\cline { 2 - 7 } Standard Deviation & 0.091039 & 0.088892 & 0.087699 & 0.087357 & $\mathbf{0 . 0 8 7 3 3 7}$ \\
\cline { 2 - 7 } Skewness & $\mathbf{0 . 3 1 9 5 7 2}$ & 0.215952 & 0.112308 & 0.050204 & 0.041352 \\
Kurtosis & 1.470049 & 1.069079 & 0.885207 & $\mathbf{0 . 8 1 7 3 5 7}$ & 0.841093 \\
Minimum & $\mathbf{- 0 . 1 9 1 2 9}$ & -0.19541 & -0.19749 & -0.19974 & -0.20228 \\
Maximum & $\mathbf{0 . 3 5 8 6 3 9}$ & 0.308329 & 0.26884 & 0.266499 & 0.267819 \\
\hline
\end{tabular}

Table 10: In-sample parameters for the return distributions of efficient portfolios in the meanvariance-0.01CVaR model with expected return $d_{5}=0.028334$. 